\title{
Le Mésolithique du Morbihan intérieur : bilan documentaire et problématique
}

The Mesolithic of inland Morbihan: evaluating data and assessing research questions

\section{Gaël Barracand}

\section{OpenEdition}

\section{Journals}

Édition électronique

URL : https://journals.openedition.org/rao/1760

DOI : $10.4000 /$ rao. 1760

ISBN : 978-2-7535-2790-4

ISSN : $1775-3732$

Éditeur

Presses universitaires de Rennes

\section{Édition imprimée}

Date de publication : 30 décembre 2012

Pagination : 25-50

ISBN : 978-2-7535-2641-9

ISSN : 0767-709X

Référence électronique

Gaël Barracand, «Le Mésolithique du Morbihan intérieur : bilan documentaire et problématique », Revue archéologique de l'Ouest [En ligne], 29 | 2012, mis en ligne le 30 décembre 2014, consulté le 24 juillet 2021. URL : http://journals.openedition.org/rao/1760 ; DOI : https://doi.org/10.4000/rao.1760

Ce document a été généré automatiquement le 24 juillet 2021.

@ Presses universitaires de Rennes 


\section{Le Mésolithique du Morbihan intérieur : bilan documentaire et problématique}

The Mesolithic of inland Morbihan: evaluating data and assessing research questions

Gaël Barracand

\section{Problématique}

1 Les études menées jusqu'à présent sur le Mésolithique dans le Morbihan s'appuient principalement sur des sites proches du littoral. Les fouilles de Téviec et de Hœdic ont notamment posé les bases des connaissances sur cette période dans la région.

2 Cette étude se place dans la continuité de plusieurs recherches effectuées sur cette période en Bretagne : par P. Gouletquer, à partir de campagnes de prospections menées dans le Finistère qui ont montré l'importance des sites préhistoriques à l'intérieur des terres (Gouletquer et al., 1997), puis par O.Kayser (1984; 1988), G. Marchand (Marchand, 1990 ; 1999) et E. Yven pour la région de Callac (Yven, 2003A ; 2003B ; 2004 ; 2006). Dans ce département, les connaissances sur le Mésolithique restent encore à préciser.

3 Cet article a pour objectif de proposer un bilan quantitatif et qualitatif des sites mésolithiques présents dans le Morbihan et de faire un point sur le Mésolithique de l'hinterland à travers l'exemple de Lande Kerroc'h au centre-nord du Morbihan, près du Blavet. Le but étant de discuter, d'une part, de la diffusion des roches et d'autre part, de l'apport des sites de surfaces.

4 Le site de Lande Kerroc'h a une particularité : la pluralité géologique de sa composition pétrographique. En effet, les sites découverts dans le Morbihan livrent des séries lithiques constituées exclusivement de silex. Celle de Lande Kerroc'h comprend des roches variées, telles que l'ultramylonite de Tréméven, le grès éocène et la calcédoine. 
Ce cortège de roches taillées est rarement présent sur un même site du premier Mésolithique. E. Yven souligne que "les stratégies d'exploitation des matières premières dépendent de la disponibilité des sources d'approvisionnement et sont donc subordonnées au fonctionnement et à la structure interne des territoires " (Yven, 2006). Ce site pourrait alimenter efficacement la question des matériaux lithiques taillables dans le Morbihan. Toutefois, la collection lithique n'étant pas homogène, il faudra être prudent sur son analyse. L'étude de cette série, qui comprend 1335 vestiges, vient compléter les informations sur le taux d'utilisation des roches locales par rapport au silex, tout en renseignant également sur le domaine de la gestion des matières premières en lien avec les contraintes physiques et que sous-tendent les territoires.

5 La composition pétrographique témoigne de réseaux de diffusion des matières premières au sein de la région. L'observation des diffusions des roches sera complétée par une étude technologique et typologique, en lien avec les matériaux utilisés, permettant d'observer leur exploitation. Ainsi, on tentera de déterminer les particularités morphologiques de roches, mises à profit pour faciliter le débitage. La mise en perspective régionale des études permet d'envisager la question des modalités d'adaptation des chasseurs-collecteurs face à leurs ressources pétrographiques en termes de ressources et de divergences.

\section{Le Morbihan}

6 La vision du Mésolithique dans le Morbihan, comme plus largement en Bretagne, a largement évolué au cours des dernières décennies. La mise en évidence de cette période dans le département est marquée par les fouilles de Téviec entre 1928 et 1930 (Péquart et al., 1937) puis d'Hœdic entre 1931 et 1934 (Péquart et al., 1954). Ces deux sites, mis au jour par Marthe et Saint-Just Péquart, présentent des groupes mésolithiques caractérisés notamment par une industrie lithique à microlithes géométriques. À partir de 1960-1974, les travaux de p. Gouletquer relancent les recherches, surtout sur le Finistère. Il est également important de noter à la fois l'influence d'Y. Largouët et plus largement de la Société polymathique du Morbihan, et celle de J.-G. Rozoy qui accentua les recherches et le travail de thèse (Rozoy, 1978) qui restent encore aujourd'hui des références en la matière. 0 . Kayser donne ensuite un cadre renouvelé au Mésolithique breton à partir de la fouille de deux niveaux coquilliers: Beg-an-Dorchenn à Plomeur (Finistère) et Beg-er-Vil à Quiberon (Morbihan). Il réalise à l'occasion un " état des connaissances » sur le Mésolithique de Bretagne (Kayser, 1991). Enfin, G. Marchand débute ses recherches sur le Mésolithique du sud de la Bretagne en y incluant le Morbihan (Marchand, 1990 ; 1992 et 1999). Les dernières études sur les amas coquilliers ont regroupé un ensemble de chercheurs qui ont développé des travaux complémentaires : C. Dupont (coquilles), Y. Gruet (crabes et balanes), N. Desse-Berset (poissons), A. Tresset (mammifères et oiseaux), R. Schulting (alimentation, datations), L. Gaudin et D. Marguerie (charbons et pollens) (Dupont et al., 2010). Les sites principalement concernés dans le Morbihan sont Téviec, Hœdic et Beger-Vil compris dans l'intervalle 6200/5000 avant notre ère. Ils s'insèrent au sein du Téviecien dans un faciès Morbihannais (Marchand, 2007). 


\section{Inventaire des sites mésolithiques dans le Morbihan}

7 Les sites présentés dans les tableaux $n^{\circ} 1, n^{\circ} 2$ et $n^{\circ} 3$ sont intégrés dans trois groupes distincts. Ils sont tous situés dans le département du Morbihan, pour la plupart près de la côte et pour certains à l'intérieur des terres, notamment le long du Blavet. Leur position est signalée sur la carte (figure $\mathrm{n}^{\circ} 1$ ).

8 Le premier groupe (tabl.1) correspond aux sites mésolithiques fouillés. Ils sont au nombre de neuf. Parmi eux, seul le site de Lannec er Gadouer est un indice de site mésolithique (trapèze à bord tronqué repris par retouches abruptes) (Cassen, 2010), les autres comprennent chacun une collection conséquente de mobilier lithique illustrant le Mésolithique.

Tableau 1 : Les sites mésolithiques du Morbihan.

Table 1: Mesolithic sites in Morbihan.

\begin{tabular}{|c|c|c|c|c|c|}
\hline $\begin{array}{l}\text { Nom des sites ou } \\
\text { EA (Entité } \\
\text { archéologique) }\end{array}$ & Commune & Période & Référence & Auteur & $\begin{array}{l}\text { Type } \\
\text { d'entité } \\
\text { (Site ou } \\
\text { Indice) }\end{array}$ \\
\hline Beg-er-Vil & Quiberon & $\begin{array}{l}\text { Mésolithique } \\
\text { final }\end{array}$ & $\begin{array}{l}\text { SRA ; Kayser et al., } \\
\text { 1988; Poissonnier } \\
\text { et Kayser } 1988 \text {; } \\
\text { Marchand, } 1999\end{array}$ & $\begin{array}{l}\text { G. Bernier et } 0 . \\
\text { Kayser }\end{array}$ & Site \\
\hline Ile Téviec & $\begin{array}{l}\text { Saint- } \\
\text { Pierre- } \\
\text { Quiberon }\end{array}$ & $\begin{array}{l}\text { Mésolithique } \\
\text { final }\end{array}$ & $\begin{array}{l}\text { SRA; Péquart et } \\
\text { al., } \\
\text { Marchand, } 2007\end{array}$ & F. Gaillard & Site \\
\hline Kerhillio & Erdeven & Mésolithique & $\begin{array}{lr}\text { Gouletquer, } \\
\text { Monnier, } & 1976 \text {; } \\
\text { Rozoy, } & 1978 \text {; } \\
\text { Kayser, } & 1984 \text {; } \\
\text { Marchand, } 2005 \mathrm{~b}\end{array}$ & F. Quatrehomme & Site \\
\hline La Croix Audran & Carnac & $\begin{array}{l}\text { Mésolithique } \\
\text { ancien-moyen }\end{array}$ & $\begin{array}{l}\text { SRA ; Blanchet, } \\
2002 ; \quad \text { Blanchet, } \\
2008\end{array}$ & S. Blanchet & Site \\
\hline $\begin{array}{l}\text { Lannec } \quad \text { er } \\
\text { Gadouer }\end{array}$ & Erdeven & $\begin{array}{l}\text { Mésolithique } \\
\text { final }\end{array}$ & SRA ; Cassen, 2000 & S. Cassen & $\begin{array}{l}\text { Indice de } \\
\text { site }\end{array}$ \\
\hline Le Dillien & Cléguerec & $\begin{array}{l}\text { Mésolithique } \\
\text { moyen, } \\
\text { Néolithique } \\
\text { ancien et final }\end{array}$ & $\begin{array}{l}\text { Marchand et al., } \\
2006\end{array}$ & $?$ & Site \\
\hline
\end{tabular}




\begin{tabular}{|c|c|c|c|c|c|}
\hline $\begin{array}{l}\text { Pointe ou Trou de } \\
\text { L'enfer }\end{array}$ & Groix & $\begin{array}{l}\text { Mésolithique } \\
\text { moyen }\end{array}$ & $\begin{array}{l}\text { SRA; Marchand, } \\
\text { 1990; Le Guen, } \\
\text { 1976; Marchand, } \\
\text { 2005b }\end{array}$ & A. Le Guen & Site \\
\hline $\begin{array}{l}\text { Port-Nehué ou } \\
\text { Hoedic }\end{array}$ & Hoedic & $\begin{array}{l}\text { Mésolithique } \\
\text { final }\end{array}$ & $\begin{array}{lr}\text { SRA ; Péquart et } \\
\text { al., } & 1954 \text {; } \\
\text { Marchand, } 2007\end{array}$ & Z. Le Rouzic & Site \\
\hline $\begin{array}{ll}\text { Terrasses } & \text { de } \\
\text { Kerjouanno } & \end{array}$ & Arzon & $\begin{array}{l}\text { Mésolithique } \\
\text { ancien-moyen }\end{array}$ & $\begin{array}{lr}\text { SRA ; } & \text { Largouet, } \\
1967 ; & \text { Rozoy, } \\
1978 ; & \text { Marchand, } \\
1990 ; & \text { Kayser, } \\
1991 & \end{array}$ & $\begin{array}{l}\text { Mr Van den } \\
\text { Broucque et P. } \\
\text { Gouletquer }\end{array}$ & Site \\
\hline
\end{tabular}

9 Le second groupe de sites (tabl.2) peut être daté du Mésolithique (du Morbihan). Ils sont issus de prospections. Ils sont au nombre de trente-et-un, Lande Kerroc'h inclus. Parmi ces sites, on note la présence de l'ultramylonite de Tréméven notamment à Guernehué (Caudan), Bourcicogne (Pont-Scorff ; Bertrand et Ginet, 2001), Lomener 1 et 2 (Pont-Scorff; Bertrand et Ginet, 2001) et Villeneuve Piriou (Guidel). Il a été observé à Lann Quehello II (Groix) de nombreux produits lamellaires et des trapèzes au Storang (Groix), dont un trapèze de Téviec. Concernant le site de la pointe de Kerzo (Austin, 1993), indiqué dans la commune de Locmaria dans l'article d'Audouard et al. (2010), il s'avère que le site est situé à Sauzon (fig. 1 ; tabl.2). De plus, ce site vient d'être redécouvert en prospection (G. Musch, communication personnelle). Le matériel mis au jour se compose d'une cinquantaine de pièces dont deux armatures. Le site du Triono (Erdeven) illustre bien la réalité du terrain et le mélange des séries de prospection qui comprennent souvent à la fois des vestiges du Mésolithique et du Néolithique (Le Guen, 1970).

Tableau 2 : Les sites mésolithiques de prospection du Morbihan

Table 2: Surveyed Mesolithic sites of Morbihan

\begin{tabular}{|c|c|c|c|c|c|}
\hline $\begin{array}{l}\text { Nom des sites } \\
\text { ou EA }\end{array}$ & Commune & Période & Référence & Auteur & $\begin{array}{l}\text { Type } \\
\text { d'entité } \\
\text { (Site ou } \\
\text { Indice) }\end{array}$ \\
\hline $\begin{array}{l}\text { Bagueneres/ } \\
\text { Bouladew }\end{array}$ & Bangor & Mésolithique & $\begin{array}{l}\text { SRA ; Batt et Kayser, } \\
1989 \text {; Audouard et al., } \\
2010\end{array}$ & O. Kayser & Site \\
\hline Beg-en-Aud & $\begin{array}{l}\text { Saint-Pierre- } \\
\text { Quiberon }\end{array}$ & Mésolithique & Kayser; 1988 & O. Kayser & Site \\
\hline
\end{tabular}




\begin{tabular}{|c|c|c|c|c|c|}
\hline Bellevue & Neulliac & $\begin{array}{l}\text { Mésolithique } \\
\text { moyen, } \\
\text { Néolithique ancien } \\
\text { et final }\end{array}$ & Marchand et al., 2006 & $?$ & Site \\
\hline Bileric-Er-Fons & Groix & $\begin{array}{l}\text { Mésolithique } \\
\text { moyen }\end{array}$ & $\begin{array}{l}\text { SRA ; Le Guen, } 1979 \text {; } \\
\text { Marchand, } 1990 \text { et } \\
1992\end{array}$ & F. Goupil & Site \\
\hline Bordelann & Sauzon & Mésolithique final & $\begin{array}{l}\text { SRA; Musch, 2004; } \\
\text { Marchand, 2009 ; } \\
\text { Audouard et al., } 2010\end{array}$ & G. Musch & Site \\
\hline Bornord & Locmaria & Mésolithique & Audouard et al., 2010 & J.-P Huot & Site \\
\hline Bourcicogne & Pont scorff & Mésolithique & $\begin{array}{l}\text { Bertrand et Ginet, } \\
2001\end{array}$ & $\begin{array}{l}\text { R. Bertand } \\
\text { et B. Ginet }\end{array}$ & Site \\
\hline Castel Dour & Cléguerec & Mésolithique & $\begin{array}{l}\text { Marchand, } \\
\text { Yven, 2001; }\end{array}$ & $?$ & Site \\
\hline $\begin{array}{l}\text { Douar } \quad \text { Port } \\
\text { Yzed }\end{array}$ & Sauzon & Mésolithique & $\begin{array}{l}\text { Batt et Kayser, } 1989 \text {; } \\
\text { et Marchand, } 1990 \text {; } \\
\text { Audouard et al., } 2010\end{array}$ & O. Kayser & Site \\
\hline $\begin{array}{l}\text { Ecluse de } \\
\text { Guernal }\end{array}$ & Neulliac & Mésolithique final & Marchand, 2001 & $\begin{array}{l}\text { G. } \\
\text { Tournay }\end{array}$ & Site \\
\hline Guernehuë & Monterblanc & $\begin{array}{l}\text { Mésolithique } \\
\text { ancien-moyen }\end{array}$ & $\begin{array}{l}\text { SRA; Lecornec, et. } \\
\text { Gouezin, 1983; } \\
\text { Marchand, } 1990\end{array}$ & J. Lecornec & Site \\
\hline Guerneüe & Caudan & Mésolithique & $\begin{array}{l}\text { Bertrand et Ginet, } \\
2001\end{array}$ & $\begin{array}{l}\text { R. Bertand } \\
\text { et B. Ginet }\end{array}$ & Site \\
\hline $\begin{array}{lr}\text { Ile } & \text { aux } \\
\text { Chevaux } & \text { ou } \\
\text { Malvant } & \end{array}$ & Ile-D'houat & Mésolithique & $\begin{array}{l}\text { SRA; Rozoy, 1978, } \\
\text { Kayser, } 1984 ; \text { BSPM } \\
\text { 19. 61-65. } 11 \text { octobre } \\
\text { 1962. Séance } 1260^{\mathrm{e}}\end{array}$ & A. Bauer & Site \\
\hline Kerlierno & Cléguerec & $\begin{array}{l}\text { Mésolithique } \\
\text { moyen et final }\end{array}$ & $\begin{array}{l}\text { Marchand,2001; Yven, } \\
2004\end{array}$ & $\begin{array}{l}\text { G. } \\
\text { Tournay }\end{array}$ & Site \\
\hline Kervin & Le Palais & $\begin{array}{l}\text { Mésolithique/ } \\
\text { Gallo-romain }\end{array}$ & $\begin{array}{l}\text { SRA; } \\
\text { Audouard et al., } 2010\end{array}$ & G. Musch & Indice \\
\hline $\begin{array}{l}\text { Lande } \\
\text { Kerroc'h }\end{array}$ & Bieuzy & $\begin{array}{l}\text { Premier } \\
\text { Mésolithique }\end{array}$ & Barracand, 2009 & S. Guérin & Site \\
\hline $\begin{array}{l}\text { Lann Quehello } \\
\text { II }\end{array}$ & Groix & Mésolithique & SRA & F.Goupil & Site \\
\hline
\end{tabular}




\begin{tabular}{|c|c|c|c|c|c|}
\hline Le Gorzed & Groix & $\begin{array}{l}\text { Mésolithique } \\
\text { moyen }\end{array}$ & Le Guen, 2007 & A. Le Guen & Site \\
\hline Le Gouello & Caudan & Mésolithique & $\begin{array}{l}\text { SRA; Bertrand, Ginet, } \\
2001\end{array}$ & B.Ginet & Site \\
\hline Le storang & Groix & Mésolithique final & SRA & F. Goupil & Site \\
\hline Le Triono & Erdeven & $\begin{array}{l}\text { Mésolithique/ } \\
\text { Néolithique }\end{array}$ & $\begin{array}{l}\text { Le Guen, } 1970 \text {; } \\
\text { Marchand, 2005b }\end{array}$ & Dr Lejards & Site \\
\hline Lomener 1 & Pont scorff & Mésolithique & $\begin{array}{l}\text { Bertrand et Ginet, } \\
2001\end{array}$ & $\begin{array}{l}\text { R. Bertand } \\
\text { et B. Ginet }\end{array}$ & Site \\
\hline Lomener 2 & Pont scorff & Mésolithique & $\begin{array}{l}\text { Bertrand et Ginet, } \\
2001\end{array}$ & $\begin{array}{l}\text { R. Bertand } \\
\text { et B. Ginet }\end{array}$ & Site \\
\hline $\begin{array}{l}\text { Moulin de } \\
\text { Kerbiquet }\end{array}$ & Guiscriff & Mésolithique & SRA ; Provost, 2006 & O. Kayser & Site \\
\hline $\begin{array}{l}\text { Pointe de } \\
\text { Kerzo }\end{array}$ & Sauzon & Mésolithique & $\begin{array}{l}\text { Austin } 1993 \text {; } \\
\text { Audouard et al., } 2010\end{array}$ & S. Austin & Site \\
\hline Pont calan & Caudan & Mésolithique & $\begin{array}{l}\text { Bertrand et Ginet, } \\
2001\end{array}$ & $\begin{array}{l}\text { R. Bertand } \\
\text { et B. Ginet }\end{array}$ & Site \\
\hline $\begin{array}{l}\text { Port des } \\
\text { sonnettes } \\
\text { (Kerledan I et } \\
\text { II) }\end{array}$ & Sauzon & Mésolithique & $\begin{array}{l}\text { Musch, 2002, } 2003 \text { et } \\
2005 \text {; Audouard et al., } \\
2010\end{array}$ & G. Musch & Site \\
\hline Port-Plouz & Ile-d'houat & Mésolithique & Kayser, 1984 & O. Kayser & Site \\
\hline Tibain & Locmaria & Mésolithique & Audouard et al., 2010 & G. Musch & Site \\
\hline Tronchâteau & Cléguer & Mésolithique & $\begin{array}{l}\text { Bertrand et Ginet, } \\
2001\end{array}$ & $\begin{array}{l}\text { R. Bertand } \\
\text { et B. Ginet }\end{array}$ & Site \\
\hline $\begin{array}{l}\text { Villeneuve } \\
\text { Piriou }\end{array}$ & Guidel & $\begin{array}{l}\text { Mésolithique/ } \\
\text { Néolithique }\end{array}$ & SRA & $\begin{array}{l}\text { R. } \\
\text { Bertrand }\end{array}$ & Site \\
\hline
\end{tabular}

10 Le troisième groupe de sites (tabl. 3) peut être daté du Mésolithique (dans le Morbihan). Toutefois, il n'est pas possible de s'en assurer : soit la bibliographie est imprécise, soit la collection n'a pu être approchée. On en dénombre dix-neuf. Le site de Kerminihy (Erdeven ; Pensec, 1973 ; Chapuy, 1975) est particulier : il comprend de très nombreux petits éclats de silex ainsi que quelques lames et lamelles brutes. L'absence de produits finis et l'abondance de produits bruts ont amené l'inventeur à caractériser ce site comme un atelier de taille. 
Tableau 3 : Les sites mésolithiques invérifiables du Morbihan.

Table 3: Unverifiable Mesolithic sites of Morbihan.

\begin{tabular}{|c|c|c|c|c|c|}
\hline $\begin{array}{l}\text { Nom des sites } \\
\text { ou EA }\end{array}$ & Commune & Période & Référence & Auteur & $\begin{array}{l}\text { Type } \\
\text { d'entité } \\
\text { (Site ou } \\
\text { Indice) }\end{array}$ \\
\hline 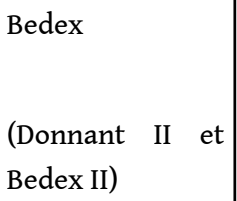 & Bangor & $\begin{array}{l}\text { Mésolithique?/ } \\
\text { Néolithique/Gallo- } \\
\text { romain }\end{array}$ & $\begin{array}{l}\text { SRA; } \\
\text { Musch, } 2002 ; \\
\text { Audouard et al., } 2010\end{array}$ & G. Musch & Site \\
\hline Castoul & Le Palais & $\begin{array}{l}\text { Mésolithique?/ } \\
\text { Néolithique }\end{array}$ & $\begin{array}{l}\text { Austin, } 1993 \text {; } \\
\text { Audouard et al., } 2010\end{array}$ & S. Austin & Site \\
\hline \begin{tabular}{ll|} 
Goah-Lêron/ & \\
village & $\mathrm{du}$ \\
Cosquer &
\end{tabular} & Plouharnel & $\begin{array}{l}\text { Mésolithique ?/Age } \\
\text { du Bronze }\end{array}$ & SRA & $\begin{array}{l}\text { F. } \\
\text { Gaillard }\end{array}$ & Site \\
\hline Kerjust & Kervignac & Mésolithique? & SRA & $\begin{array}{l}\text { R. } \\
\text { Bertrand }\end{array}$ & Site \\
\hline Kerminihy & Erdeven & $\begin{array}{l}\text { Mésolithique?/ } \\
\text { Néolithique }\end{array}$ & $\begin{array}{l}\text { Pensec, } 1973 ; \\
\text { Chapuis, } 1975\end{array}$ & $\begin{array}{l}\text { G. } \\
\text { Chapuis }\end{array}$ & Site \\
\hline Kerolland & Ile-d'Arz & $\begin{array}{l}\text { Mésolithique?/ } \\
\text { Néolithique }\end{array}$ & SRA & $\begin{array}{l}\mathrm{J}-\mathrm{Y} . \\
\text { Gallais }\end{array}$ & Site \\
\hline Kersauce I & Groix & Mésolithique? & SRA & F. Goupil & Site \\
\hline Kerroc'h & Guidel & Mésolithique? & SRA & $\begin{array}{l}\text { R. } \\
\text { Bertrand }\end{array}$ & Site \\
\hline Kervilahouen & Bangor & $\begin{array}{l}\text { Mésolithique? } \\
\text { Néolithique/Age du } \\
\text { Bronze }\end{array}$ & $\begin{array}{l}\text { SRA ; Musch, 2003; } \\
\text { Audouard et al., } 2010\end{array}$ & G. Musch & Site \\
\hline $\begin{array}{l}\text { La Côte de } \\
\text { Kerlard }\end{array}$ & Groix & Mésolithique? & SRA & F. Goupil & Site \\
\hline Le Lizo & Carnac & Mésolithique? & SRA & $\begin{array}{l}\text { A.E. } \\
\text { Riskine }\end{array}$ & Site \\
\hline
\end{tabular}




\begin{tabular}{|c|c|c|c|c|c|}
\hline Le Vieux Fort & Ploemeur & Mésolithique? & SRA & $\begin{array}{l}\text { J-M. } \\
\text { Lacot }\end{array}$ & Site \\
\hline La Villeneuve & Caudan & $\begin{array}{l}\text { Mésolithique?/ } \\
\text { Néolithique }\end{array}$ & SRA & $\begin{array}{l}\text { R. } \\
\text { Bertand }\end{array}$ & Site \\
\hline Nostang & Keryot & Mésolithique? & SRA & B. Ginet & Site \\
\hline $\begin{array}{lr}\text { Pointe } & \text { Saint } \\
\text { Colomban } & \end{array}$ & Carnac & Mésolithique? & SRA & $\begin{array}{l}\text { S. } \\
\text { Blanchet }\end{array}$ & Site \\
\hline Porcoubar & Ploemeur & Mésolithique? & SRA & $\begin{array}{l}\text { J-M. } \\
\text { Lacot }\end{array}$ & Site \\
\hline Port-Deuborch & Sauzon & Mésolithique? & $\begin{array}{l}\text { Austin } 1993 \text {; } \\
\text { Audouard et al., } 2010\end{array}$ & S. Austin & Site \\
\hline Saint Colomban & Carnac & Mésolithique? & SRA & $\begin{array}{l}\text { S. } \\
\text { Blanchet }\end{array}$ & Site \\
\hline Trebihan & Languidic & Mésolithique? & SRA & $\begin{array}{l}\text { S. } \\
\text { Blanchet }\end{array}$ & Site \\
\hline
\end{tabular}

Le Mésolithique au sein du Morbihan est illustré par cinquante-huit sites, très largement répartis sur la côte (fig. 1), qui caractérisent les différentes phases de cette période. Une partie des sites est localisée sur les îles, notamment Belle-Île-en-Mer (Austin, 1993 ; Batt et Kayser, 1989 ; Munch, 2002 ; 2003 ; 2004 ; 2005 ; Audouard et al., 2010), Groix (Le Guen, $1976 ; 1979$ et 2007). Le site du Moulin de Kerbiquet (Guiscriff) est isolé à l'ouest de l'hinterland (Provost, 2006). Malgré le nombre important de sites découverts, les informations restent insuffisantes pour bien documenter le Mésolithique de cette partie de la Bretagne.

Figure 1 : Carte des sites ou EA mésolithiques du Morbihan. DAO : G. Barracand. 
Figure 1: Map of Mesolithic sites in Morbihan.

Les sites mésolithiques dans le Morbihan (en noir) : Lande Kerroc'h, Bieuzy (étoile noire), $n^{\circ} 1$ : Bagueneres, Bangor (ou Bouladew, Bangor) ; $n^{\circ} 2$ : Beg-en-Aud, Saint-Pierre-Quiberon; $n^{\circ} 3$ : Beg-er-Vil, Quiberon; $n^{\circ} 4$ : Bellevue, Neulliac; $n^{\circ} 5$ : Bileric Er Fons, Groix; $n^{\circ} 6$ : Bordelann, Sauzon ; $n^{\circ} 7$ : Bornord, Locmaria ; $n^{\circ} 8$ : Bourcicogne, Pont-Scorff ; $n^{\circ} 9$ : Castel Dour, Cléguérec ; $n^{\circ} 10$ : Douar port yzed, Sauzon ; $n^{\circ} 11$ : écluse de Guernal, Neulliac ; $n^{\circ} 12$ : Guernehue, Monterblanc ; n 13 : Guernehué, Pont-Scorff ; $n^{\circ} 14$ : île Téviec, Saint-Pierre-Quiberon ; $n^{\circ} 15$ : île aux Chevaux ou Malvant, île d'Houat ; n 16 : Kerhillio, Erdeven ; n 17 : Kerlierno, Cléguérec ; n 18 : Kervin, Le Palais ; n 19 : La Croix-Audran, Carnac ; $n^{\circ} 20$ : Lannec er Gadouer, Erdeven ; $n^{\circ} 21$ : Lann Quehello II, Groix ; $n^{\circ} 22$ : Le Dillien, Cléguérec ; n 23 : Le Gorzed, Groix; n² 24 : Le Gouello, Caudan ; n 25 : Le Storang, Groix; n 26 : Le Triono, Erdeven ; $n^{\circ} 27$ : Lomener 1 et Lomener 2, Pont-Scorff ; $n^{\circ} 28$ : Moulin de Kerbiquet, Guiscriff ; $n^{\circ} 29$ : Pointe de l'Enfer, Groix; $n^{\circ} 30$ : Pont Calan, Pont-Scorff; n 31 : Port-Les-Sonnettes (Kerledan 1 et 2), Sauzon; $n^{\circ} 32$ : Port-Nehué ou Hœdic, Hœdic ; $n^{\circ} 33$ : Port-Plouz, île d'Houat ; $n^{\circ} 34$ : Terrasses de Kerjouanno, Arzon; $n^{\circ} 35$ : Tibain, Locmaria ; $n^{\circ} 36$ : Tronchâteau, Cléguérec ; $n^{\circ} 37$ : Villeneuve Piriou, Guidel ; $n^{\circ} 38$ : Pointe de Kerzo, Sauzon. $n^{\circ} 39$ : Castoul, Le Palais ; $n^{\circ} 40$ : Trebihan, Languidic ; $n^{\circ} 41$ : Saint Colomban, Carnac ; $n^{\circ} 42$ : Goah-Lêron/village du Cosquer, Plouharnel ; $n^{\circ} 43$ : Port Deuborch, Sauzon ; $n^{\circ} 44$ : Porcoubar, Ploemeur ; $n^{\circ} 45$ : Kerjust, Kervignac ; $n^{\circ} 46$ : Kerminihy, Erdeven ; $n^{\circ} 47$ : Kerolland, île d'Artz ; $n^{\circ} 48$ : Kersauce I, Groix ; $n^{\circ} 49$ : Kerroc'h, Guidel ; $n^{\circ} 50$ : Kervazic, Erdeven; $n^{\circ} 51$ : Kervilahouen, Bangor ; $n^{\circ} 52$ : la côte de Kerlard, Groix; $n^{\circ} 53$ : La Villeneuve, Caudan; $n^{\circ} 54$ : Bedex, Bangor (Donnant II et Bedex II) ; $n^{\circ} 55$ : pointe Saint-Colomban, Carnac ; $n^{\circ} 56$ : Nostang, Keryot ; $n^{\circ} 57$ : Le Lizo, Carnac ; n 58 : Le Vieux Fort, Ploemeur.

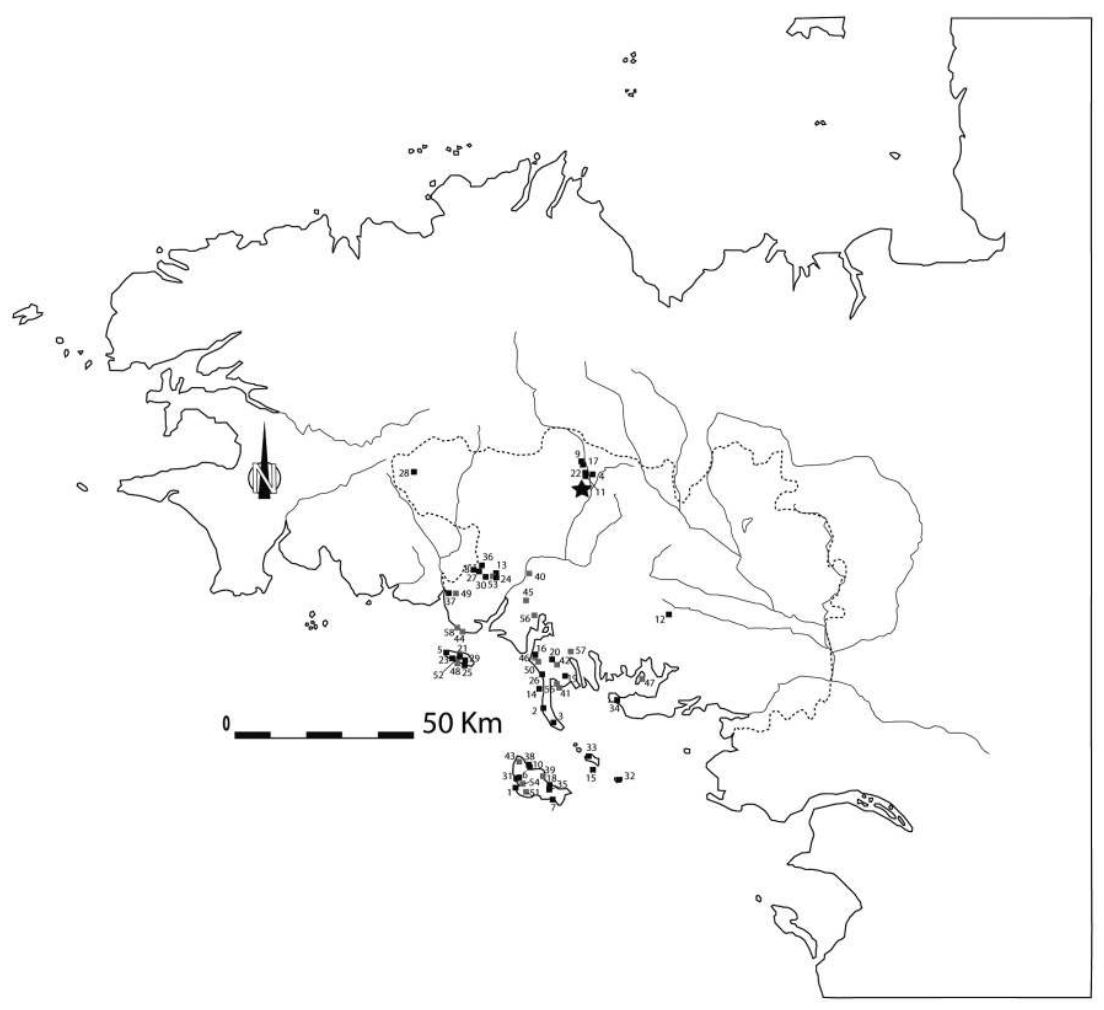

\section{Attribution chrono-culturelle}

Le Mésolithique en Morbihan est intégré dans le groupe sud-breton selon J.-G Rozoy (1978) auquel succèderait le Téviecien.

Pour le premier Mésolithique du Morbihan, on peut évoquer les sites de Guernehué (Monterblanc), Er-Fons/Biléric (Groix) et Kerjouann ${ }^{\circ}$ (Arzon; Kayser, 1991; Marchand, 1992; Tsobgou Ahoupe, 2007). Ils correspondent à un stade ancien/moyen et présentent un assemblage microlithique constitué de triangles scalènes et isocèles accompagnés de pointes à base concave. Le débitage de style Coincy est présent ainsi que les micro-burins. 

représenté. Quelques sites ont été reconnus ces dernières années (Castel Dour, Cléguérec; Bellevue, Neulliac; Écluse de Guernal ; Kerlierno, Cléguérec; Le Dillien, Cléguérec; Moulin de Kerbiquet, Guiscriff). Au centre du département, une concentration de sites se dessine de part et d'autre du Blavet, témoignant de l'importance de ce fleuve pour les chasseurs-collecteurs de l'époque. À ce groupe, vient s'ajouter le site de Lande Kerroc'h (Barracand, 2009) dont la collection est composée d'un corpus comprenant une vingtaine de roches bretonnes à extrarégionales.

\section{Les sites de prospections}

Étant majoritaires par rapport aux sites fouillés pour le Mésolithique dans le Morbihan, il convient d'énoncer quelques points :

- Les collections sont parcellaires. Les vestiges sont dégagés, souvent suites aux travaux agricoles dont les stigmates rendent parfois difficile la distinction entre retouches liées à l'utilisation ou à la fabrication.

- Bien que de petits éléments soient souvent récoltés, leur nombre est en-deçà de la réalité et la série d'armatures est aussi amoindrie. Ce constat s'applique probablement à Lande Kerroc'h.

18 C'est pourquoi les éléments les plus représentatifs à considérer face à ces collections restent les nucléus et les outils qui, malgré les chocs subis, conservent des informations qu'il convient d'exploiter. Pour la Bretagne, ce genre de collection comporte aussi un intérêt dans l'hinterland pour la représentation de l'exploitation des différents types de roches. Les nucléus et les outils permettent, lorsque la série est suffisante, d'estimer les objectifs de la production et les supports. Ils servent en outre à proposer un cadre chronologique souvent large. En complément, l'analyse des produits bruts apporte des éléments complémentaires quant aux choix et styles de débitage.

\section{Contexte du site de Lande Kerroc'h}

19 Le site de Lande Kerroc'h ( $\mathrm{X}=0201,019$ et $\mathrm{Y}=2347,555$; fig. 2 ) a été découvert en prospection par Stéphane Guérin en 2004. Il est localisé en bord de plateau de la vallée 
du Blavet, en position dominante. La collection a été constituée suite à un ramassage de surface, et la question de sa représentativité et de sa valeur statistique restent entières.

Figure 2 : Localisation du site de Lande Kerroc'h. DAO : G. Barracand.

Figure 2: Location of Lande Kerroc'h site.

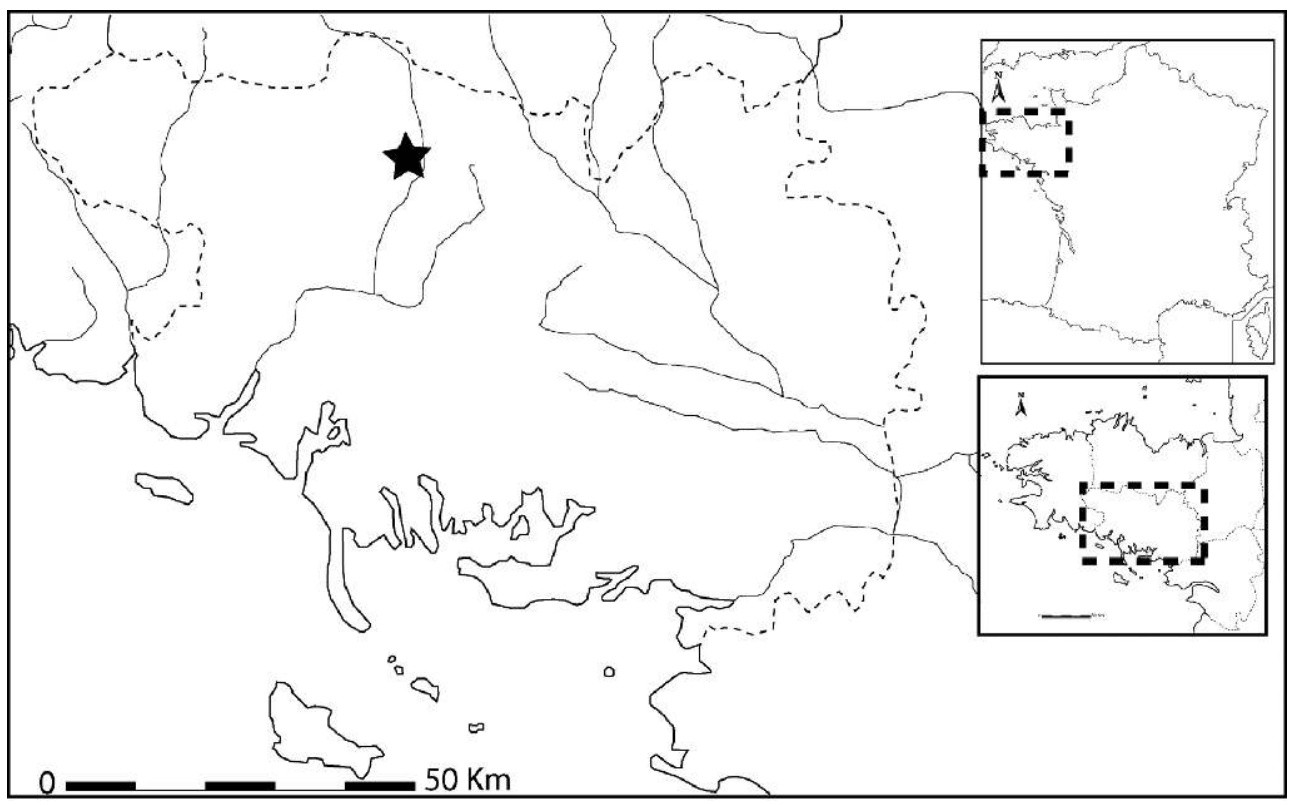

Répartition géologique du matériel lithique

La détermination des roches (tabl. 4) a été supervisée par R. Tsobgou Ahoupe. Le silex pris dans son ensemble représente un peu plus de $50 \%$ du corpus total des roches suivi par les grès éocènes $(18,7 \%)$ et l'ultramylonite de Tréméven $(12,7 \%)$. Le silex est composé de rognons provenant sans doute des côtes. Quelques silex du Jurassique $(1,6 \%)$ et du Crétacé $(0,7 \%)$ à cortex natif le complètent. Le premier peut provenir de Sarthe ou de Normandie et le second peut-être de la vallée du Loir ou de la Seine. On observe aussi une infime présence de galets alluviaux, ainsi que trois galets indéterminés.

Tableau 4 : Représentation générale du corpus lithique par roche avec leur effectif et le pourcentage correspondant.

Table 4: General representation of the lithic corpus by rock with total number and percentage.

\begin{tabular}{|l|l|l|}
\hline Résultats & $\begin{array}{l}\text { Nombres de pièces par type de } \\
\text { roche }\end{array}$ & $\begin{array}{l}\text { \% de pièces par type de } \\
\text { roche }\end{array}$ \\
\hline $\begin{array}{l}\text { Noms des différentes } \\
\text { roches }\end{array}$ & 1335 & $\mathbf{1 0 0 \%}$ \\
\hline Silex & 699 & 52,4 \\
\hline Grès & 249 & 18,7 \\
\hline
\end{tabular}




\begin{tabular}{|l|l|l|}
\hline Ultramylonite de Tréméven & 168 & 12,9 \\
\hline Calcédoine & 69 & 5,2 \\
\hline Phtanites de Callac & 38 & 2,9 \\
\hline Roches indéterminées & 35 & 2,6 \\
\hline Silcrètes & 28 & 2,1 \\
\hline Quartz filonien & 23 & 1,7 \\
\hline Cataclasite de Mikaël & 9 & 0,7 \\
\hline Microquartzites & 9 & 0,7 \\
\hline Quartzite & 4 & 0,3 \\
\hline Dolérite & 1 & 0,1 \\
\hline Gneiss & 1 & 0,1 \\
\hline Quartz cristal & 1 & 0,1 \\
\hline Quartzarénite de Montbert & 1 & 0,1 \\
\hline Phtanites de Lamballe & 1 & 0,1 \\
\hline
\end{tabular}

\section{Les gisements et la géologie des roches}

21 Aucun gisement géologique n'est à proximité immédiate (fig. 3). Il est intéressant de noter que ces gisements sont pour certains à plus de cinquante kilomètres du site (fig. 3), alors qu'«il ne semble pas y avoir d'échanges de roches métamorphiques locales entre le sud et le nord de la péninsule " (Marchand et Tsobgou Ahoupe, 2007). Même si différents éléments chronologiques peuvent se superposer, quelques originalités se dégagent.

Figure 3 : Zone potentielle d'affleurement des roches découvertes à Lande Kerroc'h (FL : ForestLanderneau). D'après une carte géologique de R. Tsobgou Ahoupe, 2007, modifiée ; DAO :

G. Barracand. 
Figure 3: Outcrop area of rocks discovered at Lande Kerroc'h.

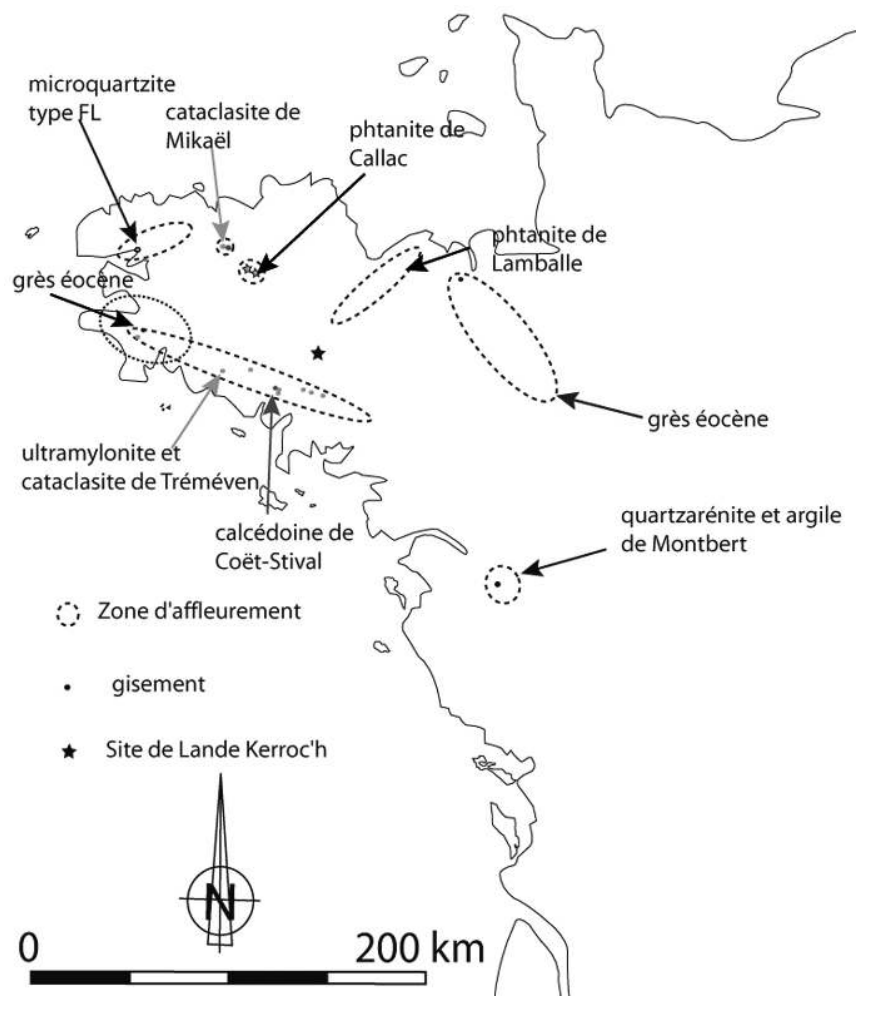

\section{Les roches sédimentaires}

Sur le site, tous les types de silex - la calcédoine, les phtanites, les silcrètes, le grès et la quartzarénite de Montbert - font partie de ce groupe. Le silex proviendrait logiquement de la côte située à environ quarante kilomètres au sud du Morbihan. Ils apparaissent pour l'essentiel sous la forme de galets littoraux (silex marin; Hallegouët et al., 1980). Mais on note des silex avec un cortex plus lustré (silex alluvial), différent de celui dit marin. La calcédoine a été signalée par Gérard Tournay à Coët-Stival (Languidic). Au Mésolithique, les phtanites proviennent pour la plupart de Callac (Dabard, 1997 ; Yven, 2003a ; 2003b ; 2004 ; 2006 ; Tsobgou Ahoupe, 2007). Ceux du site semblent venir de Callac, sauf un de Lamballe comportant une pigmentation noire et présentant une granulation polymorphe dispersée entre les cristaux de quartz qui dessinent la lamination dans le cas des faciès laminés de Lamballe. Les silcrètes pourraient provenir de la surface du Massif armoricain, où elles ont été découvertes pour la première fois (Estéoule-Choux, 1981 et 1983). Les grès éocènes s'observent surtout dans des zones de dépressions. Le gisement le plus connu est celui de Kervouster (Béchennec et al., 1999). Enfin la quartzarénite de Montbert est un grès dont la taille des grains appartient à la classe des arénites (Tsobgou Ahoupe, 2007).

\section{Les roches métamorphiques}

On comptabilise l'ultramylonite de Tréméven, la cataclasite de Mikaël, le quartz filonien, le gneiss, le quartzite et le microquartzite. L'ultramylonite en Bretagne provient de faciès sombres, dérivés de formations en minéraux basiques (Jégouzo et Rossollo, 1988 ; Tsobgou Ahoupe, 2007). Celle du site provient sans doute de la zone de Languidic à vingt kilomètres au sud. Les gisements de cataclasite de Mikaël observés 
sont situés à plus de soixante kilomètres du site, au nord du cisaillement armoricain (Tsobgou Ahoupe, 2007). Le quartz se rencontre au sein des failles sous la forme de filons (quartz filonien) ou en amas de cristaux automorphes (cristal de roche). À l'inverse des quartzites constitués de macro-cristaux, les microquartzites sont principalement constitués de microcristaux de quartz. Ceux dits armoricains dérivent de grès paléozoïques. Ils ont été découverts pour la première fois à la ForestLanderneau (Chauris et Hallegouët, 1973 ; Tsobgou Ahoupe, 2007).

La dolérite est une roche magmatique, massive et compacte, le plus souvent vert sombre. Elle est employée ici pour la réalisation d'un galet biseauté.

Il est intéressant de voir que l'assemblage lithique de Lande Kerroc'h est composé de roches (les cataclasites de Mikaël) qui pourraient provenir de plus de soixante kilomètres, et de certains silex (silex jurassiques et crétacés) acquis peut-être en dehors de la Bretagne. D'une part, ceci témoignerait de déplacements ou d'échanges sur de grandes distances ; d'autre part, cela attesterait que le Massif armoricain est traversé et que des déplacements de roches métamorphiques locales entre le sud et le nord de la péninsule se seraient déroulés.

\section{Présentation de la collection lithique}

Les principales roches (tabl. 5) sont dans l'ordre d'importance : le silex (52,4 \%), les grès éocènes $(18,7 \%)$ et l'ultramylonite de Tréméven $(12,6 \%)$. Cet ordre marque un attachement au silex et une adaptation aux matières premières de l'hinterland. La calcédoine est la moins présente des roches principales $(5,2 \%)$. Les éléments thermiques sont nombreux: $42,3 \%$ au sein du silex, $0,6 \%$ parmi les pièces en ultramylonite de Tréméven et $14,5 \%$ au sein du grès, ce qui témoigne de la mise en œuvre de foyers. Les roches indéterminées présentent $57,1 \%$ de pièces thermiques. Cependant, les éléments thermiques en silex représentent $80 \%$ du total des pièces thermiques. Toutes ces pièces ont subi une chauffe accidentelle, indiquant une proximité avec des foyers. La série comprend $79,1 \%$ de produits bruts, $15,2 \%$ d'outils et $7,6 \%$ de nucléus. On observe une forte présence d'éclats (1056), des lames (73) et lamelles (77) viennent compléter la collection. Le taux de nucléus (20,3\%) est important parmi la calcédoine qui ne comporte que peu de pièces. On note un taux important d'outils $(14,8 \%)$ au sein du grès. La composante lithique en silex pour les outils est identique au grés éocène et 7,6\% de nucléus sont présents.

Tableau 5 : Synthèse du corpus de Lande Kerroc'h.

Table 5: Lande Kerroc'h assemblage synthesis.

\begin{tabular}{|l|l|l|l|l|l|l|l|l|}
\hline & $\begin{array}{l}\text { Débitage } \\
\text { retouché- } \\
\text { utilisé et } \\
\text { brut }\end{array}$ & $\begin{array}{l}\text { les outils } \\
\text { et } \\
\text { armatures }\end{array}$ & Nucleus & Total & $\begin{array}{l}\text { Pièces } \\
\text { thermiques }\end{array}$ & $\begin{array}{l}\text { Eclats } \\
\text { entiers et } \\
\text { fragments }\end{array}$ & $\begin{array}{l}\text { Lames } \\
\text { entières } \\
\text { et } \\
\text { fragments }\end{array}$ & $\begin{array}{l}\text { Lamelles } \\
\text { entières } \\
\text { et } \\
\text { fragments }\end{array}$ \\
\hline Silex & 532 & 104 & 53 & 699 & 296 & 549 & 36 & 48 \\
\hline
\end{tabular}




\begin{tabular}{|c|c|c|c|c|c|c|c|c|}
\hline Grès éocène & 188 & 37 & 8 & 249 & 36 & 217 & 11 & 14 \\
\hline $\begin{array}{l}\text { Ultramylonite } \\
\text { de Tréméven }\end{array}$ & 112 & 38 & 17 & 168 & 1 & 117 & 22 & 4 \\
\hline Calcédoine & 50 & 5 & 14 & 69 & 7 & 50 & 1 & 2 \\
\hline $\begin{array}{l}\text { Phtanite de } \\
\text { Callac }\end{array}$ & 31 & 4 & 3 & 38 & 0 & 31 & 2 & 2 \\
\hline $\begin{array}{l}\text { Roche } \\
\text { indéterminée }\end{array}$ & 32 & 2 & 1 & 35 & 20 & 29 & 0 & 4 \\
\hline Silcrète & 18 & 7 & 3 & 28 & 9 & 23 & 0 & 2 \\
\hline $\begin{array}{l}\text { Quartz } \\
\text { filonien }\end{array}$ & 21 & 2 & 0 & 23 & 0 & 20 & 0 & 1 \\
\hline $\begin{array}{l}\text { Cataclasite de } \\
\text { Mikaël }\end{array}$ & 9 & 0 & 0 & 9 & 0 & 9 & 0 & 0 \\
\hline Microquartzite & 7 & 1 & 1 & 9 & 1 & 6 & 1 & 0 \\
\hline Quartzite & 3 & 1 & 0 & 4 & 0 & 3 & 0 & 0 \\
\hline Dolérite & 0 & 1 & 0 & 1 & 0 & 0 & 0 & 0 \\
\hline Gneiss & 1 & 0 & 0 & 1 & 0 & 1 & 0 & 0 \\
\hline Quartz cristal & 1 & 0 & 0 & 1 & 0 & 1 & 0 & 0 \\
\hline $\begin{array}{l}\text { Quartzarénite } \\
\text { de Montbert }\end{array}$ & 0 & 1 & 0 & 1 & 0 & 0 & 0 & 0 \\
\hline $\begin{array}{l}\text { Phtanite de } \\
\text { Lamballe }\end{array}$ & 0 & 0 & 1 & 1 & 0 & 0 & 0 & 0 \\
\hline Totaux & 1005 & 203 & 101 & 1335 & 370 & 1056 & 73 & 77 \\
\hline
\end{tabular}

La présence d'une vingtaine de roches au sein de la collection lithique constituée à $50 \%$ de silex peut s'expliquer par l'éloignement important entre le site et les zones à silex. L'analyse des gîtes de matières premières a montré que certaines roches pourraient provenir d'aussi loin, voire plus, que le silex qui reste la roche la plus présente au sein du corpus. Bien que la collection lithique du site de Lande Kerroc'h soit incomplète le pourcentage faible de ces roches en provenance lointaine permet de s'interroger sur la signification de ces roches. Étant donné que les qualités des roches (les cataclasites de Mikaël et les phtanites) à la taille sont médiocres, avec en général une production de produits épais, leur intérêt pourrait alors être autre que clastiques. Symbolique? Économique? Mais elles pourraient être simplement le reflet d'une mode ou d'une facilité d'approvisionnement témoignant alors de la vitalité des déplacements des roches en Bretagne. 


\section{Le débitage}

\section{Les produits de débitage}

Seules les roches caractérisées par un effectif de pièces en nombre suffisant peuvent être abordées sous l'angle de leurs modalités de transformations.

La prépondérance de la production d'éclats, toutes roches confondues, témoigne d'une mise en forme sur place puisqu'elle accompagne différentes étapes du débitage amenant à la réalisation d'outils, y compris la mise en forme. Cela signifie donc que les blocs sont arrivés bruts ou à peine dégrossis. La facilité étant le transport de blocs destinés à être débités pour obtenir différents types de supports.

L'observation des produits des trois principales roches montre des variantes dans la présence de produits laminaires et lamellaires. Le silex présente un plus fort taux de lamelles brutes (32) par rapport aux lames (15) alors que pour les grès éocènes, les taux sont équivalents (7). L'ultramylonite de Tréméven diffère avec un plus fort taux de lames brutes (11) que de lamelles brutes (4). L'examen des éclats montre une standardisation équivalente au sein de ces trois roches (Barracand, 2009).

31 La percussion directe dure est manifeste par la proéminence des bulbes et la présence de talons lisses minces ou larges sur les produits corticaux.

\section{Les nucléus}

Les 101 nucléus collectés offrent une image (tabl.6) des schémas techniques mis en œuvre sur le site de Lande Kerroc'h, mais à l'instar des éclats, le lien avec les lamelles reste difficile. On compte environ un nucléus pour treize éclats. Parmi ceux-ci, huit ont été sélectionnés et dessinés (cinq en silex, deux en ultramylonite et un en grès éocène) pour observer plus précisément les modalités de débitage.

33 La calcédoine concentre le plus fort pourcentage de nucléus par rapport à son nombre total de pièces $(20,3 \%)$. Cela pourrait indiquer un débitage des produits sur place et le transport des outils vers un autre lieu.

Tableau 6 : Les nucléus du site de Lande Kerroc'h et leur production.

Table 6: Lande Kerroc'h site cores production.

\begin{tabular}{|c|c|c|c|c|c|c|c|c|c|c|}
\hline $\begin{array}{l}\text { Mode de } \\
\text { débitage }\end{array}$ & Silex & Calcédoine & Grès & $\begin{array}{l}\text { Phtanite } \\
\text { de } \\
\text { Callac }\end{array}$ & $\begin{array}{l}\text { Phtanite } \\
\text { de } \\
\text { Lamballe }\end{array}$ & Silcrète & Microquartzite & Indéterminé & Ultramylonite & Total \\
\hline $\begin{array}{l}\text { Bipolaire } \\
\text { unifacial }\end{array}$ & 3 & 2 & & & & 1 & & & 1 & 7 \\
\hline $\begin{array}{l}\text { Bipolaire } \\
\text { bifacial }\end{array}$ & 3 & 1 & 1 & & & & 1 & & 4 & 10 \\
\hline $\begin{array}{l}\text { Percussion } \\
\text { bipolaire } \\
\text { sur enclume }\end{array}$ & 1 & & & & & & & & & 1 \\
\hline
\end{tabular}




\begin{tabular}{|c|c|c|c|c|c|c|c|c|c|c|}
\hline $\begin{array}{l}\text { Bipolaire } \\
\text { alterne }\end{array}$ & 3 & & & & & & & & & 3 \\
\hline $\begin{array}{l}\text { Unipolaire } \\
\text { unifacial }\end{array}$ & 2 & 2 & & 1 & & & & & 2 & 7 \\
\hline $\begin{array}{l}\text { Unipolaire } \\
\text { bifacial }\end{array}$ & 2 & 2 & & & & & & & 3 & 7 \\
\hline $\begin{array}{l}\text { Unipolaire } \\
\text { tournant }\end{array}$ & 10 & & 3 & 1 & & 1 & & & 2 & 17 \\
\hline $\begin{array}{ll}\text { Front de } \\
\text { débitage }\end{array}$ & 1 & & & & & & & & & 1 \\
\hline $\begin{array}{l}\text { Débitage } \\
\text { centripète }\end{array}$ & 3 & 1 & 2 & & & & & & & 6 \\
\hline Multipolaire & 3 & 1 & & & 1 & & & & 4 & 9 \\
\hline $\begin{array}{l}\text { Unipolaire } \\
\text { semi- } \\
\text { tournant }\end{array}$ & 9 & 1 & 1 & 1 & & 1 & & & & 13 \\
\hline Indéterminé & 9 & 1 & & & & & & 1 & & 11 \\
\hline $\begin{array}{l}\text { Bipolaire } \\
\text { multifacial }\end{array}$ & & 1 & & & & & & & & 1 \\
\hline $\begin{array}{l}\text { Nucleus mis } \\
\text { en forme }\end{array}$ & 2 & & & & & & & & & 2 \\
\hline $\begin{array}{l}\text { Bipolaire } \\
\text { tournant }\end{array}$ & 2 & 2 & 1 & & & & & & & 5 \\
\hline $\begin{array}{l}\text { Unipolaire } \\
\text { préférentiel }\end{array}$ & & & & & & & & & 1 & 1 \\
\hline Production & & & & & & & & & & \\
\hline Eclats & 19 & 8 & 5 & 2 & 1 & 1 & 1 & & 5 & 42 \\
\hline $\begin{array}{l}\text { Eclats } \quad+ \\
\text { lames }\end{array}$ & 1 & & & & & & & & 2 & 3 \\
\hline $\begin{array}{l}\text { Eclats } \quad+ \\
\text { lamelles }\end{array}$ & 5 & 5 & 3 & & & 2 & & & 10 & 25 \\
\hline Lamelles & 20 & & & 1 & & & & & & 21 \\
\hline Indéterminé & 8 & 1 & & & & & & 1 & & 10 \\
\hline Total & 53 & 14 & 8 & 3 & 1 & 3 & 1 & 1 & 17 & 101 \\
\hline
\end{tabular}


première analyse permet de voir que trente nucléus montrent une gestion du débitage unipolaire tournante ou semi-tournante. Cela concerne, parmi les principales roches, dix-neuf sur cinquante-trois nucléus en silex, deux sur dix-sept en ultramylonite et quatre sur huit en grès. Pour l'ultramylonite, les principaux modes opératoires sont bipolaires bifaciaux et multipolaires, comportant chacun quatre éléments. La production observée à partir des négatifs d'enlèvements est majoritairement à éclats pour quarante-deux nucléus, puis à éclats et lamelles (25), enfin à lamelles (21). La lamelle reste le produit de prédilection. Les éclats montrent des négatifs de lamelles témoignant de leur recherche. La caractérisation des nucléus par un débitage, notamment unipolaire semi-tournant, rappelle le Mésolithique moyen de Bretagne occidentale (Yven, 2006). Cependant, si les éclats sont aussi multiformes, les lamelles ici sont plus grandes.

Les nucléus sélectionnés illustrent les observations générales. La production unipolaire tournante à éclats est illustrée par le nucléus en grès, de taille réduite qui montre une utilisation optimale de la matière et une volonté de débiter de tout petits produits (fig. $5 n^{\circ}$ 6) et le nucléus en silex (fig. $6 n^{\circ} 7$ ) donne une idée de la dimension initiale des galets, car il garde encore une grande partie du cortex. Il présente des négatifs d'éclats plus larges. On observe en étape A et A' quelques micro-arrachements qui ont servi à nettoyer le plan de frappe. La production unipolaire tournante et semi-tournante à éclats et lamelles ou lamelles est illustrée par six nucléus. Le nucléus en ultramylonite (fig. $4, \mathrm{n}^{\circ} 1$ ) présente une production à éclats et à lamelles unipolaire unifaciale avec des rythmes 1-2-3 permettant de couvrir toute la surface. Cela s'explique par le fait que le débitage est sur un éclat sui sert à l'obtention de lamelles, les éclats ayant pour but la correction de la carène. Le nucléus (fig. $6, \mathrm{n}^{\circ}$ 8) en silex est unipolaire tournant, avec une production à éclats et lamelles. Le nucléus (fig. $4, n^{\circ} 2$ ) en silex a été exploité par un débitage unipolaire semi-tournant à lamelles avec des rythmes 1-2-3. On note, sur la face inférieure, une tentative d'ouverture d'un second front de débitage. Le nucléus $\mathrm{n}^{\circ}$ 5 (fig. 5) est le plus petit de toute la collection. Il est unipolaire tournant a production lamellaire. Le débitage se fait en trois étapes et les réaménagements sont parallèles. Le nucléus $n^{\circ} 3$ (fig. 4) est bipolaire unifacial sur lamelles en silex. Les deux plans de frappe ont servi au détachement de lamelles. Le nucléus (fig. $5, \mathrm{n}^{\circ} 4$ ) est unipolaire préférentiel à lames puis éclats. Ce nucléus en ultramylonite présente en phase $\mathrm{A}$ un débitage d'éclats et en phase B un débitage de grandes lames en utilisant le plan de foliation de la roche. Cet exemplaire montre que le tailleur avait une bonne connaissance de la structure de l'ultramylonite favorable au détachement des lames. On observe ce comportement au sein de certaines industries du second Mésolithique où l'ultramylonite est fortement exploitée (Creac'h Miné Vihan ; Tsobgou Ahoupe, 2006).

Figure 4 : Nucléus de Lande Kerroc'h. DAO : G. Barracand. 
Figure 4: Lande Kerroc'h cores.

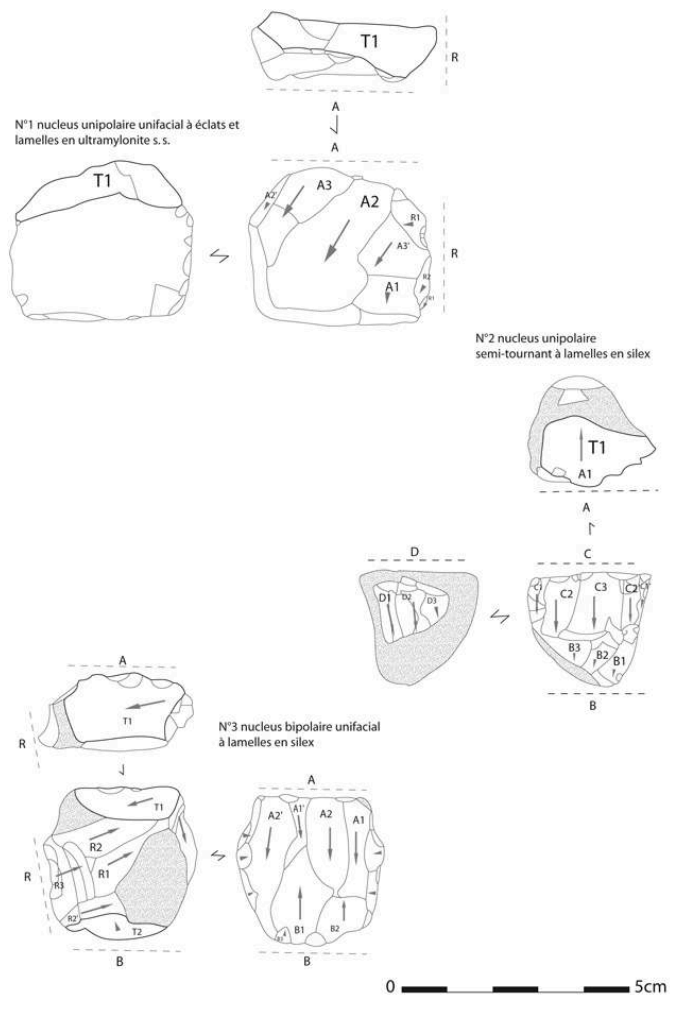

Figure 5 : Nucléus de Lande Kerroc'h. DAO : G. Barracand.

Figure 5: Lande Kerroc'h cores.

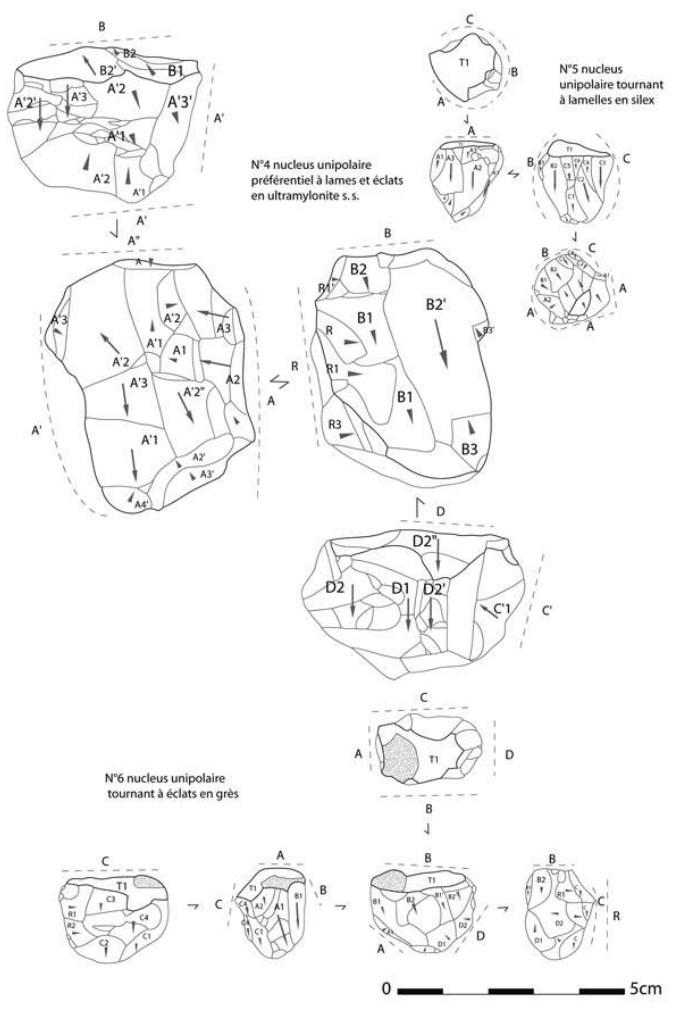


Figure 6: Lande Kerroc'h cores.

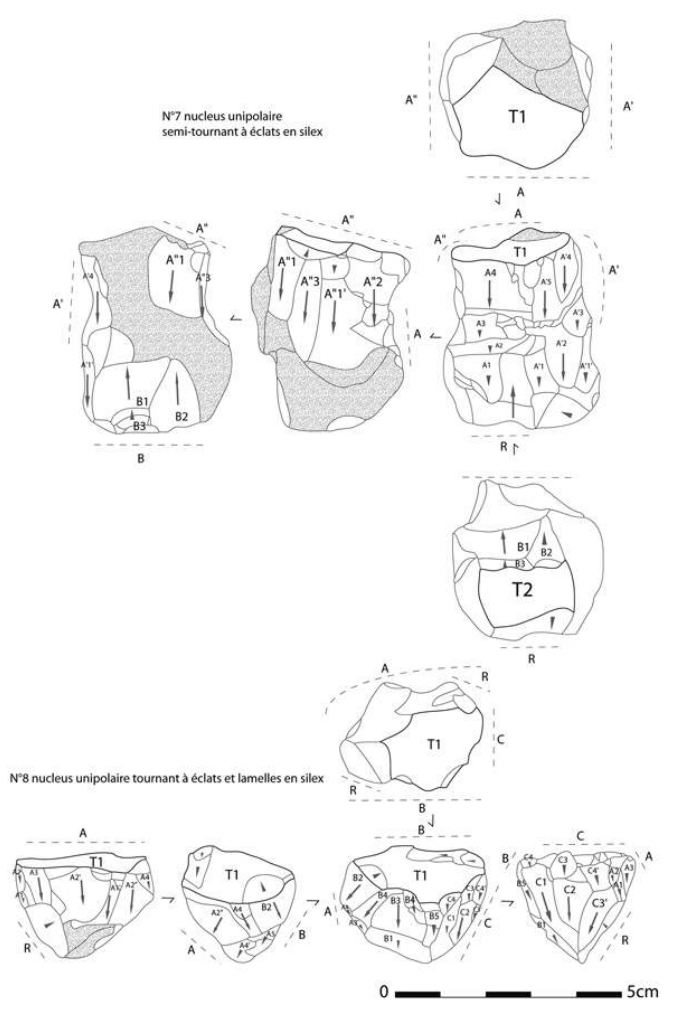

La présence d'un éclat de percussion bipolaire sur enclume est à noter à Lande Kerroc'h. On retrouve quelques traces de ce type de percussion au premier Mésolithique à Biléric et au Trou de l'enfer (île de Groix, Morbihan) et au second Mésolithique à Beg-er-Vil (Quiberon, Morbihan). Son développement ne se fait réellement qu'au Néolithique (Guyodo et Marchand, 2005). Le mélange des périodes au sein du site ne permet pas plus de précisions sur ce vestige.

Les éclats d'avivage observés (fig. $7, \mathrm{n}^{\circ} 13$ ) témoignent des réaménagements observés sur les nucléus. L'entretien des flancs est réalisé par des enlèvements d'éclats ou de lamelles suivant un plan orthogonal ou sécant. Trois néo-crêtes en silex (fig. $8, \mathrm{n}^{\circ} 3$ et $n^{\circ} 7$ ) attestent de remises en forme au cours du débitage. Trois tablettes, dont deux en silex et une en ultramylonite (fig. 8, $n^{\circ} 8$ ), renseignent sur les techniques de renouvellement de plan de frappe pour continuer le débitage. Deux éclats outrepassés en silex (fig. $7, n^{\circ} 15$ ) correspondent à un enlèvement important de la partie distale, dont une partie distale en corticale.

Figure 7 : Outillage de Lande Kerroc'h. DAO : G. Barracand.

Figure 7: Lande Kerroc'h tools.

$n^{\circ} 1-2$ : armatures en cours de fabrication; $n^{\circ} 3$ : distal de lame à dos; $n^{\circ} 5$ : lamelle à dos ; $n^{\circ} 4-6$ fragment de lamelle à dos; $n^{\circ} 7:$ pointe; $n^{\circ} 8:$ trapèze rectangle en grès; $n^{\circ} 9:$ fragment de lamelle à coche, dit micro-burin ; $n^{\circ} 10$ : éclat tronqué ; $n^{\circ} 11$ : lamelle tronquée; $n^{\circ} 12$ : fragment de tronqué ; $n^{\circ} 13$ : éclat de ravivage $; n^{\circ} 14$ : lame tronquée; $n^{\circ} 15$ : fragment d'éclat outrepassé. 


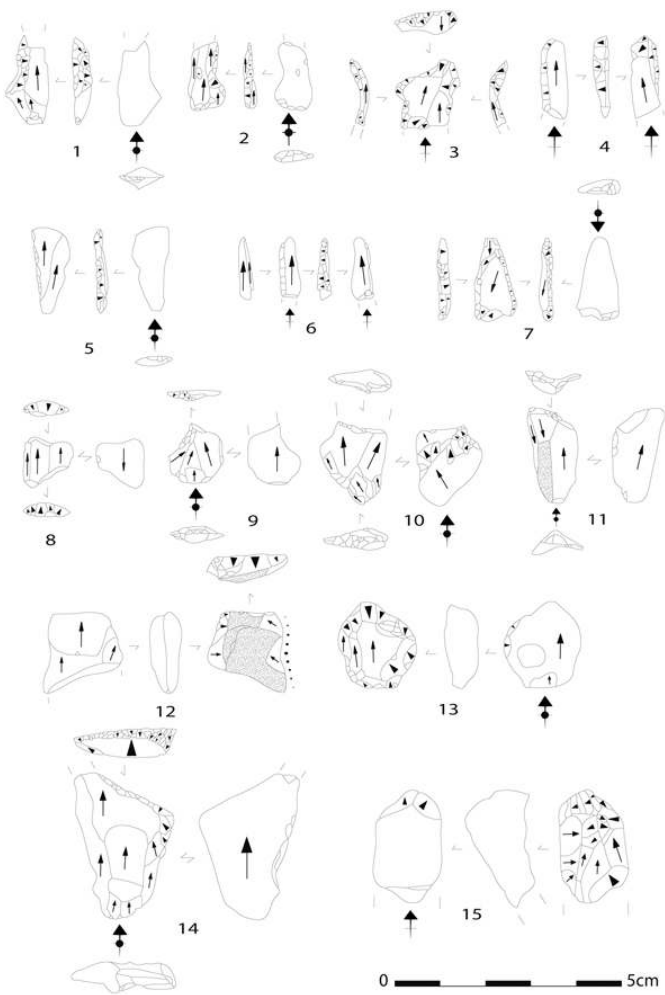

Figure 8 : Outillage de Lande Kerroc'h. DAO : G. Barracand.

\section{Figure 8: Lande Kerroc'h tools.}

$N^{\circ} 1-2$ : grattoirs; $n^{\circ} 3$ : fragment de néo-crêtes; $n^{\circ} 4$ : perçoir ; $n^{\circ} 5$ : pièce esquillée ; $n^{\circ} 6$ : burin ; $n^{\circ} 7$ : fragment de néo-crêtes; $n^{\circ} 8$ : fragment de tablette de ravivage; $n^{\circ} 9$ : lame à double troncature

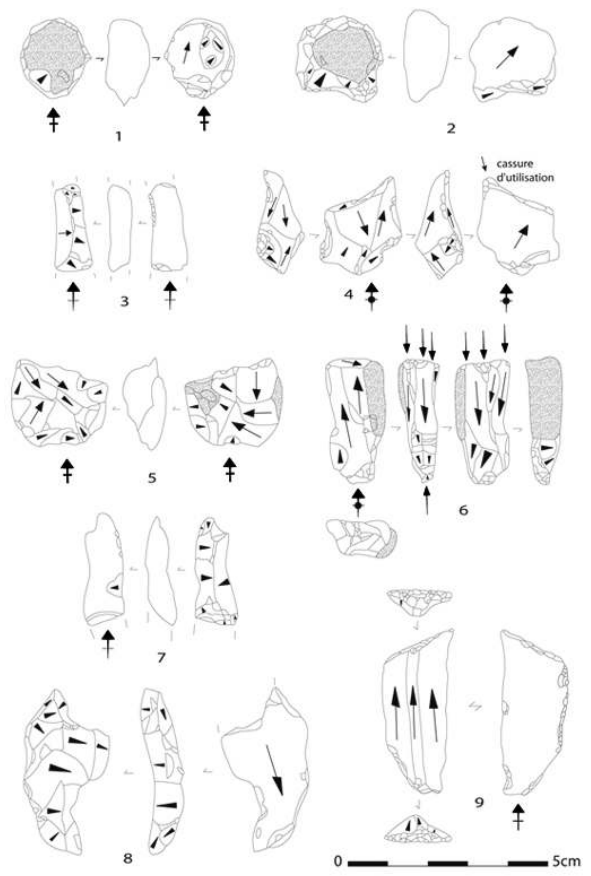



débitage sur place. Ne comportant pas de richesse géologique particulière, il est remarquable de constater un apport aussi éclectique de matière première au travers de plusieurs phases d'occupation. Les vecteurs de déplacement des roches témoignent d'un réseau convergent vers un lieu d'activités particulières (chasse, et/ou échange, agrégation?). La présence de ces nombreuses roches pourrait témoigner aussi de contacts et de l'existence de réseaux sociaux. Enfin, la forte présence du silex, même éloignée des zones de gisements, n'est pas négligeable et pourrait indiquer une préférence culturelle qui valorise cette roche comme on peut l'observer plus à l'ouest de la Bretagne au Mésolithique moyen (Yven, 2006).

\section{Les outils}

41 Ils comprennent deux cent trois éléments dont cinquante-huit outils (tabl. 7). Parmi les outils retouchés, seuls les supports lamellaires (5) sont en silex à l'exception d'une roche indéterminée. Quelques lames (3) sont en ultramylonite et en grés éocène, quatorze en silex et une en phtanite de Callac. Enfin, la part des éclats, quelles que soient les roches, est encore prépondérante. La thèse de pièces retouchées par utilisation étant douteuse du fait du labour et de la patine, elles sont laissées de côté. Le groupe des coches et des grattoirs (fig. 9, $n^{\circ} 1$ et $n^{\circ} 3$ ) domine et met en œuvre, en majorité, des éclats. La pièce $n^{\circ} 9$ (fig. 7) est un micro-burin témoignant de la fabrication d'armatures. Suivent des denticulés, des racloirs (fig. $9, \mathrm{n}^{\circ} 2$ ), des pièces esquillées (fig. $8, n^{\circ} 5$ ) et burins (fig. 8, $n^{\circ} 6$ ) fabriqués aussi en majorité sur éclats. Les perçoirs (fig. $8, n^{\circ} 4$ ) et pièces tronquées (fig. $7, n^{\circ} 10, n^{\circ} 11, n^{\circ} 12, n^{\circ} 14$ ) sont fabriqués plutôt sur lames. Quelques autres outils particuliers apparaissent au sein de cette collection (Barracand, 2009). 
Tableau 7 : Les outils et armatures de Lande Kerroc'h.

Table 7: Lande Kerroc'h tools and weapons.

\begin{tabular}{|l|l|}
\hline Types d'outils et armatures & Total \\
\hline Outils & \\
\hline Outils à coche & 14 \\
\hline Grattoirs & 10 \\
\hline Outils denticulés & 8 \\
\hline Outils tronqués & 5 \\
\hline Lame et lamelles à dos & 4 \\
\hline Racloirs & 3 \\
\hline Pièces esquillées & 3 \\
\hline Perçoirs & 2 \\
\hline Armatures en cours de fabrication & 2 \\
\hline Burins & 1 \\
\hline Galet à retouches bifaciales & 1 \\
\hline Galet biseauté & 1 \\
\hline Petit tranchet & 1 \\
\hline Lissoir & 1 \\
\hline Trapèze & 1 \\
\hline Pointe & 1 \\
\hline Total & 1 \\
\hline
\end{tabular}

Figure 9 : Outillage de Lande Kerroc'h. DAO : G. Barracand.

Figure 9: Lande Kerroc'h tools.

$n^{\circ} 1$ : éclat à coche ; $n^{\circ} 2$ : racloir ; $n^{\circ} 3$ : grattoir.

42 Le groupe des armatures comprend huit éléments. Les éléments les plus anciens seraient les lamelles à dos. Les pièces à dos sont caractéristiques du carquois du premier Mésolithique et sont un élément de détermination attribuable peut-être au Mésolithique moyen du Morbihan (fig. 7, $n^{\circ}$ 4-5-6 ; fig. 7, $n^{\circ} 3$ ). Deux armatures en cours 
de fabrication sont inachevées (fig. $7, \mathrm{n}^{\circ} 2$ et $\mathrm{n}^{\circ} 1$ ) et sont de même facture ; seul le bord retouché change. Ces deux produits sont assez irréguliers et il semble qu'ils aient été destinés à la conception de triangle. Le trapèze (fig. $\left.7, n^{\circ} 8\right)$ est en grès, contrairement aux autres en silex. Ce trapèze asymétrique est conçu à partir d'une lamelle large $(11 \mathrm{~mm})$ et très régulière à trois pans. Cet élément géométrique est un trapèze rectangle correspondant au second Mésolithique. L'armature en forme de pointe (fig. 7, $\mathrm{n}^{\circ} 7$ ) semble correspondre au type du premier Mésolithique.

\section{En résumé}

Le corpus lithique de Lande Kerroc'h tend à montrer pour le silex, un débitage passant par la mise en forme de gros éclats de décorticage et une production d'éclats destinés à la réalisation de grattoirs, racloirs et coches principalement. En parallèle, une production d'éclats allongés, de lames et de lamelles destinée à la fabrication d'armatures, de burins et de pièces tronquées a été observée. En ce qui concerne le grès et l'ultramylonite, on constate l'existence de produits de mise en forme de même style. La production d'outils diffère. Le grès est concerné par une production de supports plus courte que le silex et des outils sur éclats parmi les coches, grattoirs et denticulés. L'unique trapèze de la collection et les lamelles à dos témoignent d'outils sur lamelles. L'ultramylonite comporte d'une part un outillage sur éclats dont les coches, racloirs, grattoirs et perçoirs et d'autre part, un outillage spécifique sur lame pour les denticulés, lié à l'utilisation du plan de foliation pour la production de grandes lames. La collection lithique de Lande Kerroc'h n'est pas homogène et témoigne de plusieurs passages caractérisés par des époques et des cultures différentes éloignées dans le temps.

- Le silex $(52,4 \%)$, le grès $(18,7 \%)$ et l'ultramylonite de Tréméven $(12,6 \%)$ sont les roches majoritaires.

- La préparation des nucléus explique la présence de produits détachés par percussion directe dure, tandis que le plein débitage est caractérisé par une technique assez tendre.

- Les aménagements passent par le détachement d'éclats orthogonaux ou sécants. Le débitage implique rarement une préparation, parfois présente par des micro-arrachements (abrasion de corniche).

- L'outillage est varié, dominé par les coches et les grattoirs.

- Les schémas de débitage sont unipolaires semi-tournants et unipolaires tournants avec production d'éclats et de lamelles. Le rythme prépondérant est 1-2-3.

\section{Synthèse de l'étude}

Cinquante-huit sites ou EA (Lande Kerroc'h compris) dits chronologiquement du Mésolithique ont été dénombrés en Morbihan. Leur attribution n'est assurée que pour une partie des sites, comme nous l'avons expliqué auparavant. Ils se situent en grande majorité sur la côte, comme l'illustre la figure $\mathrm{n}^{\circ} 1$. Cela montre l'attraction de l'océan, pour ses ressources alimentaires, de même que la pétrographie avec la proximité du silex - matière première la plus utilisée. Ceci rappelle cependant que les prospections se concentrent sur la côte. Malgré cela, quelques découvertes de sites mésolithiques sont à signaler à l'intérieur des terres et notamment le long du Blavet où se situe Lande Kerroc'h. On peut évoquer (fig. 1) une nouvelle fois l'attrait de l'eau. Une chaîne de sites se dessine dans l'hinterland (fig. 1, sites $\mathrm{n}^{\circ} 12,40,36,13,24,8,27,45,30,53,28$ ). Si les 
recherches étaient accentuées dans les terres, celles-ci pourraient, à l'avenir, rejoindre le groupe de sites au nord du Morbihan et modifier cette vision du Mésolithique, centrée sur l'espace maritime. Il serait intéressant de cibler les prochaines recherches sur le Mésolithique du Morbihan vers l'intérieur des terres et dans la partie est du département afin de combler le grand vide visible au travers de la figure $\mathrm{n}^{\circ} 1$, grâce à des campagnes de prospections, puis le cas échéant à des fouilles ou tout du moins à des sondages.

\section{Le Morbihan}

Comparons maintenant le site de Lande Kerroc'h aux autres sites mésolithiques au sein du Morbihan. Celui-ci illustre bien le phénomène des sites mésolithiques de l'intérieur des terres comme le site voisin, Kerlierno (Cléguérec; fig. 1). Ce dernier, appartenant au Mésolithique moyen et final (Marchand, 2001) est situé sur une basse terrasse du Blavet au nord de Lande Kerroc'h. Il comprend un ensemble de roches (silex, quartz, grès, quartzite, phtanite, calcédoine, microquartzite, jaspe, ultramylonite de Tréméven, cornaline et meulière) en partie présentes à Lande Kerroc'h. Le débitage est initié par le détachement de produits épais à la percussion dure. Il existe trois types de production : autonome à éclats, progression faciale ou frontale, et production à lamelles fines et éclats (Yven, 2004). On peut corréler cette dernière information avec une partie de la production de Lande Kerroc'h qui témoigne d'une chaîne opératoire intégrée à éclats et lamelles au Mésolithique moyen. Kerlierno apparaît comme un site frère de Lande Kerroc'h par sa proximité, sa concentration en roches multiples et ses schémas de débitage similaires. À partir de ces deux sites, on peut exposer un profil caractéristique de l'intérieur des terres, à savoir, la diversité des roches utilisées et la présence modérée du silex (52,4\% Lande Kerroc'h ; 69,04\% Kerlierno) qui s'explique par une situation géographique éloignée des zones côtières. Le silex reste malgré tout majoritaire; il correspond à un choix prépondérant malgré la distance d'acquisition éloignée $(40 \mathrm{~km})$ et la faible dimension des nodules à exploiter. Sa qualité clastique semble donc être un critère de sélection. Si on se rapproche de la côte, on s'aperçoit d'une augmentation notable de la présence du silex au sein des matériaux exploités, comme en témoigne le site de Guernehué (Monterblanc; fig. 1). Celui-ci, éloigné de seulement vingt kilomètre de la côte ( $40 \mathrm{~km}$ pour Lande Kerroc'h), comprend au sein de sa collection $98,7 \%$ de silex. Ce site du Mésolithique ancien/moyen occupe un sommet en bord de plateau dans les Landes de Lanvaux (Lecornec et Gouezin, 1983). Comme à Lande Kerroc'h, mais en moindre quantité, on retrouve une variété de matériaux (silex, jaspe, quartzite, grès et quartz). Le débitage est assez semblable, car majoritairement unipolaire avec l'obtention de lamelles courtes régulières, mais les armatures divergent, marquées plus par des pointes et des triangles. Le micro-burin est attesté et la production de lames est importante. Si la diversité des matériaux n'est pas la même, les techniques de débitages semblent, elles, être similaires. Le site de Guernehué est un témoin mésolithique localisé dans le centre-est du département et traduit un potentiel de zones périphériques à exploiter.

Après les témoins mésolithiques proches, intéressons-nous aux sites mésolithiques fouillés dans le Morbihan. Kerjouann (Arzon; fig. 1) confirme la tendance d'une forte présence du silex $(93,22 \%)$ en provenance de l'estran. Ce site, référence du groupe sudbreton de J.-G Rozoy, est situé sur une pointe rocheuse à l'extrémité de la presqu'île de Rhuys (Largouët, 1967 ; Gouletquer et Monnier, 1976 ; Rozoy, 1978 ; Marchand, 1990). 
Les matières premières y sont variées (silex, quartz, grès, granit). Le débitage de type Coincy selon J.-G. Rozoy, est représenté par des lamelles courtes aux arêtes sinueuses. Les armatures sont caractérisées par les triangles scalènes plaidant pour une phase moyenne tandis que les trapèzes évoquent une phase plus récente. La technique du micro-burin est avérée. Kerjouann ${ }^{\circ}$ semble bien loin de Lande Kerroc'h de par sa distance $(40 \mathrm{~km})$ et son outillage. Les seuls liens pour l'outillage seraient les denticulés et un trapèze asymétrique, ce qui semble insuffisant. La présence de plusieurs phases du Mésolithique est aussi effective à Lande Kerroc'h. La Croix-Audran (Carnac; fig. 1) récemment fouillé en 2001 a été découvert dans le cadre de la construction d'une déviation routière visant à mettre en valeur les alignements de Carnac (Blanchet, 2002 ; 2008). Ce site comprend une production de supports allongés privilégiée et destinée à la fabrication d'armatures. Comme à Lande Kerroc'h, les nucléus témoignent en majorité de débitages unipolaires semi-tournants avec une production lamellaire. L'outillage est marqué par les triangles scalènes puis isocèles, le reste étant des pointes, des trapèzes symétriques et des outils communs. La Croix-Audran et Lande Kerroc'h sont à rapprocher pour ce qui concerne la base des nucléus et leur production, mais se différencient par l'outillage. Beg-er-Vil (Quiberon; fig. 1) est un amas coquillier et l'un des piliers de l'analyse des industries du Mésolithique final breton (Kayser et Bernier, 1988). Le silex, majoritaire, est accompagné par des galets de quartz, des grès et du quartzite. Le rythme de débitage frontal selon l'agencement 2-1-2' est prééminent (Marchand, 1999). Les nucléus sont en général unipolaires avec une production laminolamellaire et une autre d'éclats. L'outillage comprend en majorité des outils utilisés, des coches, des denticulés et des troncatures. Les armatures sont dominées par les trapèzes symétriques. Le micro-burin est absent. Beg-er-Vil semble marquer un écart chronologique avec Lande Kerroc'h. En effet, même si la production lamellaire est commune, les armatures ne le sont pas. Ces dernières témoignent d'étapes différentes du Mésolithique. Téviec, (Saint-Pierre-Quiberon), site éponyme du Téviecien, est situé en contexte insulaire. Celui-ci est marqué par la production de trapèzes dont le type dit de Téviec. L'essentiel de l'outillage taillé est composé de lames à troncature oblique (ou couteaux), grattoirs, éclats denticulés, trapèzes et triangles (Péquart et al., 1937 ; Rozoy, 1978). Lande Kerroc'h se différencie de ce site et du groupe auquel il appartient. En effet, il s'intègre plutôt dans un groupe plus ancien marqué par des armatures à dos et une diversité des matériaux exploités liée à l'éloignement de la côte. Même s'il apparaît évident que l'apport de sites fouillés est de toute autre nature que celui des séries de surface, ces dernières présentent un certain potentiel d'étude et autorisent des comparaisons.

\section{Les Côtes-d'Armor}

Traversons maintenant les hauteurs du Massif armoricain pour observer la région de Callac dans les Côtes-d'Armor. Cette région est intéressante par sa position à l'intérieur des terres et la présence de gisements de phtanite, source de matière première lithique exploitée à Lande Kerroc'h. Ces gisements de phtanite semblent avoir été utilisés par une série de sites comme source d'approvisionnement (Keristen 1 et 2, Kerrunet 2 à Carnoët, Kerannou à Plusquellec; fig. 10), implantés notamment dans le secteur d'un versant de la vallée de l'Hyère ou implantés sur un interfluve dominant la vallée du Blavet (Kergoubleau à Saint-Nicolas-du-Pélem; fig. 10 ; Yven, 2004). Ces sites ont été découverts en prospection et sont à composante mésolithique ancien/moyen. Les séries 
sont dominées par le phtanite suivi par le silex. Les nucléus en phtanite examinés sur les sites de la région de Callac sont exploités de même manière que les exemplaires en silex. Parmi les pièces en phtanite, on observe une épaisseur importante des produits, constat identique à Lande Kerroc'h. En revanche, sur ce dernier, celles en silex sont deux fois moins épaisses. On note aussi avec le phtanite, une production plus tournée vers l'éclat comme à Lande Kerroc'h. E. Yven remarque que les outils en phtanite et en silex sont typologiquement semblables avec toutefois, pour les individus en phtanite, des mensurations plus variées et un caractère plus massif. Le silex semble avoir été privilégié pour la production de lamelles et de microlithes alors que « le phtanite local aurait suffi à effectuer tous les objets nécessaires de l'outillage commun aux microlithes " (Yven, 2004). Les collections des sites de la région de Callac suggèrent une complémentarité dans l'utilisation du phtanite et du silex. À Lande Kerroc'h, l'absence d'outils en phtanite ne permet pas une telle conclusion mais la proximité des roches et des sites pourrait traduire une même complémentarité dans l'emploi des matériaux.

Figure 10 : Carte des sites mésolithiques de Bretagne cités. DAO : G. Barracand.

étoile noire : Lande Kerroc'h, Bieuzy ; $n^{\circ} 1$ : Keristen 2, Carnoët ; $n^{\circ} 2$ : Keristen 1, Carnoët ; $n^{\circ} 3$ Kerrunet 2, Carnoët; $n^{\circ} 4$ : Kerannou, Plusquellec; $n^{\circ} 5$ Kergoubleau, Saint-Nicolas-du-Pélem; $n^{\circ} 6$ : Creac'h Miné Vihan, Saint-Thurien; $n^{\circ} 7$ : La Villeneuve, Locunolé ; $n^{\circ} 8$ : La Presqu'île, Brennilis.

Figure 10: Map of Mesolithic sites in Brittany.

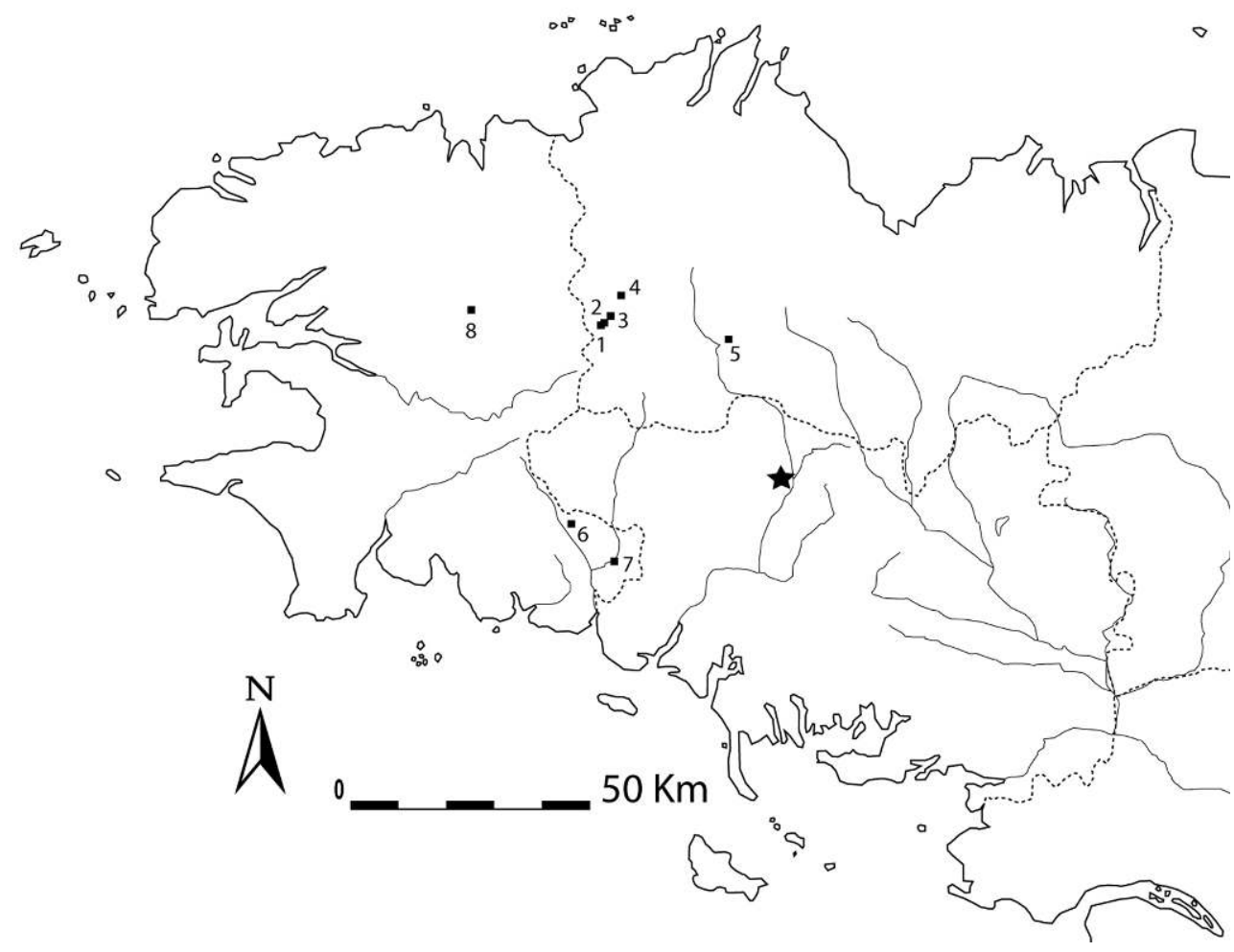

Bien que ces sites soient situés dans l'hinterland, comme celui de Lande Kerroc'h, on remarque pour ce dernier, un silex majoritaire malgré le fait que les gisements soient éloignés. La présence de chasseurs-collecteurs dans cette zone ne peut donc pas s'expliquer par l'existence de gisements de matière première proches. Qu'est-ce qui a donc attiré les hommes sur la zone de Lande Kerroc'h? L'implantation du site à proximité du Blavet, une situation dominante sur la zone avec, sans doute, la présence 
d'animaux proches, pourrait en partie expliquer les passages de chasseurs-collecteurs sur ce site.

\section{Le Finistère}

49 Terminons ce tour d'horizon des sites mésolithiques bretons par le Finistère. $\mathrm{Ce}$ département est celui sur lequel ont été effectuées les recherches les plus approfondies pour la période, grâce, notamment, aux campagnes de prospections initiées par p. Gouletquer dans les années soixante-dix. On dénombre actuellement (actualisation décembre 2009) quatre-vingt-quatorze sites rattachés au Mésolithique dans le Finistère (on prend en compte les ensembles comportant plus de trente pièces; Marchand, 2005a). Ce panel de sites a permis de mettre en évidence que «le mode d'occupation mésolithique le plus fréquent à l'extrémité de la péninsule armoricaine était un habitat de l'intérieur et non pas un amas coquillier» (Marchand, 2005a). Pour nos comparaisons entre Lande Kerroc'h et les sites du Finistère, nous choisissons des sites fouillés qui ont un lien soit géographique et technologique soit une présence de matière première similaire. À cet effet, Creac'h Miné Vihan (Saint-Thurien; fig. 10) positionné dans le Sud-Finistère sur un lieu majeur d'exploitation d'ultramylonite/cataclasite en Bretagne, de culture téviecienne, présente un intérêt particulier (Tsobgou Ahoupe, 2006 ; 2007). Parce qu'il a fait l'objet d'une étude similaire à la nôtre, il constitue un point de comparaison très pertinent. Il comprend des matières premières variées (silex, ultramylonite, cataclasite, phtanite, grès, quartz, quartzite, gneiss, calcaire). Creac'h Miné Vihan présente, comme à Lande Kerroc'h, la production d'éclats associée à des lames et lamelles. Le débitage unipolaire se retrouve mais le multipolaire y est plus important qu'à Lande Kerroc'h. L'outillage est dominé par les pièces utilisées, suivies par les coches, mais les armatures divergent par la présence de trapèzes. Pour l'ultramylonite, on observe la même utilisation des plans de foliation de cette roche (se référer à l'étude des nucléus de ce même article). Le site de La Villeneuve à Locunolé (fig. 10) est placé à cinq cents mètres d'un filon d'ultramylonite, visible dans une falaise de l'Ellé et est positionné à vingt kilomètres de la côte. Pourtant, quand on observe la série du sondage 2001, on remarque que le silex est présent à plus de $88 \%$ et l'ultramylonite à 5,3\% seulement. Si on compare ce phénomène aux sites de la Vallée de Callac, on constate que la position côtière (source de silex) détermine une forte présence du silex, même si une autre roche est disponible en abondance à proximité du site. Le rôle joué par l'exploitation des gisements diminue donc à mesure que les sites se rapprochent de la côte. La meilleure aptitude du silex à la taille apparaît comme discriminante. Sa présence au sein des collections lithiques semble ainsi être fonction de la distance à parcourir pour s'en procurer, fonction de la présence d'autres roches à proximité et fonction des objectifs du débitage. Quant à la présence de l'ultramylonite, elle semble perdre de son importance sur ce site malgré la proximité des gisements: situation différente à Lande Kerroc'h avec des gisements connus plus éloignés et où, toutefois, l'ultramylonite reste bien présente. À Creac'h Miné Vihan, cette roche est prépondérante et les gisements sont proches. L'utilisation de cette roche apparaît donc variable en fonction de l'intérêt de chacun des groupes de chasseurs-collecteurs. L'occupation du Mésolithique moyen sur ce site appartient au groupe de Bertheaume caractérisé par des lamelles étroites à un ou deux bords abattus, tronquées ou non, par des triangles scalènes étroits et par des pointes étroites à un bord abattu, avec ou sans 
base. Ce groupe du Mésolithique moyen est donc clairement différent du Mésolithique moyen que l'on trouve dans le Morbihan et notamment à Lande Kerroc'h.

Le site de la presqu'île à Brennilis (fig. 10) semble intéressant en raison de ses points communs avec Lande Kerroc'h: tout d'abord, sa position à l'intérieur des terres; ensuite, la diversité des matériaux présents au sein de la collection lithique et enfin, la présence majoritaire du silex et associé à l'ultramylonite. Ce site comprend une proportion des matériaux contraire à "la règle de décroissance fréquente à cette époque, dans laquelle les matériaux les plus proches sont les plus abondants" (Marchand, 2005a). L'ultramylonite est largement représentée par de petits éclats contrairement à Lande Kerroc'h où ceux-ci sont en moyenne plus nombreux que ceux en silex. Le silex de Brennilis comprend deux fois plus de produits lamino-lamellaires $(24,47 \%)$ qu'à Lande Kerroc'h $(12,01 \%)$. Les lamelles de Brennilis sont régulières et à deux et trois pans, obtenues à partir d'un débitage à la percussion indirecte. Elles semblent plus récentes que celles de Lande Kerroc'h. La forte présence de trapèzes symétriques à Brennilis indiquerait des phases du Mésolithique sur ces deux sites, Brennilis semblant plus récent. Seule une lamelle à dos se rapproche de celles trouvées à Lande Kerroc'h, signalant une présence plus ancienne. L'exploitation des nucléus diffère. Alors qu'à Lande Kerroc'h, on observe plutôt un débitage trifacial, à Brennilis, celui-ci est bifacial. La production de lamelles et d'éclats minces pour le silex est privilégiée, ce qui ne diffère pas trop de ce que l'on observe à Lande Kerroc'h. Enfin, le déplacement des phtanites de Callac ne répondant pas à un besoin technique à Brennilis, témoigne tout de même d'une dynamique des économies mésolithiques qui pourraient se refléter à Lande Kerroc'h.

\section{Conclusion et perspectives}

51 Lande Kerroc'h correspond, par sa composition géologique élargie, à un site de l'hinterland, avec une proportion de silex représentée à moitié alors que les roches locales sont plus importantes sur les sites côtiers. La représentativité de matières est le reflet d'une économie locale à régionale du premier Mésolithique, mais caractérise un territoire d'acquisition restreint au Mésolithique final. Le corpus lithique semble correspondre pour une bonne part a ce que l'on conçoit du Mésolithique du Morbihan avec la présence de lamelles à dos, un débitage unipolaire tournant, avec une production de lamelles et des éclats de remise en forme. Les éléments en nombre important de denticulés et grattoirs constituent l'outillage de ce premier Mésolithique $\mathrm{du}$ Morbihan. Un trapèze rectangle et l'exploitation relativement importante du grés éocène attesteraient peut-être de traces plus récentes du Mésolithique. Un grattoir anguiforme, une lame à double troncature et des perçoirs pourraient être liés à une présence néolithique.

Le Blavet semble concentrer un groupe de l'hinterland. Ainsi, ces groupes du premier Mésolithique voyagent de leur lieu de vie à la mer et en ramènent les produits. Ce groupe de l'hinterland échangerait avec le nord de la Bretagne, des roches pouvant attester ces mouvements économiques, et ce qui conduit à une interactivité entre le nord et le sud de la péninsule. Ces transports de roches sont le reflet de groupes diachroniques, ce qui amoindrit la densité de ce flux économique mais soulève quand même le problème du rassemblement de toutes ces économies sur ce même lieu, qui plus est, sur un laps de temps aussi grand. Le premier Mésolithique du Morbihan 
semble s'inscrire dans un système de relations multi-scalaires de niveau local et régional et avec des relations intergroupes en Bretagne. C'est ce qu'illustrent la diversité des matériaux présents à Lande Kerroc'h et la mise en perspective avec d'autres sites régionaux. Le bilan réalisé à partir des sites mésolithiques du Morbihan soulève de nombreuses pistes de réflexion dont celle sur les matières premières de l'hinterland bien que l'appartenance chrono-culturelle des sites reste à préciser.

À tous ceux qui m'ont aidé dans la réalisation de cet article: Grégor Marchand, Stéphane Guérin, Rodrigue Tosbgou Ahoupe, Nicolas Naudinot, Sylvène Michel, Klet Donnart, Yoann Chantreau, Briagell Huet, Marie-Dominique Pinel, Thierry Lorho, Léa Gourio, Gérald Musch, Hélène Pioffet, Marc-James Randerson, Armelle Gilois, Gilles Barracand.

\section{RÉSUMÉS}

Des prospections réalisées dans la commune de Bieuzy ont permis de mettre au jour une collection composée de vingt types de roches dont l'ultramylonite dite de Tréméven et le grès éocène. Certaines roches semblent être originaires d'au moins soixante kilomètres du site. L'éloignement de la côte explique le pourcentage réduit de silex. Les vestiges lithiques de Lande Kerroc'h témoignent d'adaptations locales $d u$ débitage en fonction des ressources pétrographiques. Ce type de site découvert en prospections, majoritaire dans la région pour la période concernée ici, permet d'engager une réflexion sur le type d'étude à faire à de tels contextes. L'inventaire des sites mésolithiques du Morbihan amène à faire le point sur cette période et prépare le terrain pour de nouvelles recherches à venir.

An inventory work of Mesolithic sites in Morbihan enabled a report on the last hunthergatherers in this area. Sites are listed in tables and maps. They are discussed with reference to the lithic assemblage from the hinterland site of Lande Kerroc'h. A survey based in Bieuzy village allowed the study of a heterogeneous assemblage made up of twenty different types of rock among which was the ultramylonite "of Tréméven" and eocene sandstone. Some rocks seem to be from at least $60 \mathrm{kms}$ from the site. The remoteness from the coast is a clue to understanding the low flint percentage. Lithic remains of Lande Kerroc'h testify of local adaptation, in order to deal with geological substratum debitage. The surveyed sites, typical in the area for the period, will allow the development of a sensible study, suitable for such archaeological contexts. Helped by the inventory of these Mesolithic sites of Morbihan département, one can evaluate compiled information upon the period and can equally prepare the ground for new research to come.

\section{INDEX}

Keywords : first Mesolithic, ultramylonite, Blavet, debitage economy, assessment, Morbihan Mots-clés : premier Mésolithique, ultramylonite, Blavet, économies, bilan, Morbihan 
AUTEUR

GAËL BARRACAND

Inrap, 1 Allée Adèle de Bretagne, 35200 Rennes, gael.barracand@gmail.com 